\title{
Seismotectonic analysis around the Mont Terri rock laboratory (Switzerland): a pilot study
}

\author{
Martinus Abednego $^{1} \cdot$ Patrick Blascheck $^{2} \cdot$ Senecio Schefer $^{3} \cdot$ Christophe Nussbaum $^{3} \cdot$ \\ Manfred Joswig $^{2} \cdot$ Paul Bossart ${ }^{3} \cdot$ Jon Mosar $^{1}$
}

\begin{abstract}
For this pilot study we used recorded seismic events from the SED permanent network and data from a dedicated SNS network to improve the seismotectonic understanding of very weak seismicity in the vicinity of the Mont Terri underground laboratory. We combined field data on faults with microseismic events and modelling of stress and focal mechanisms. Eighty-six events with very low magnitudes $(\mathrm{ML} \approx-2.0$ to 2.0 ) recorded between July 2014 and August 2015 were located within a radius of $10 \mathrm{~km}$ of the underground laboratory and used for modelling. We compiled 234 fault/striation data from laboratory tunnels and regional geology, and also from seismic/borehole data on basement faults. With this database we defined seven groups of main faults in the cover and four groups in the basement. For each of these groups we computed a synthetic focal mechanism that was subsequently used to determine a synthetic P-phase waveform. The synthetic waveforms were then correlated with the microseismic events of the cover and the basement respectively. Of these, 78 events yielded satisfactorily correlation coefficients that we used for a regional
\end{abstract}

$\triangle$ Martinus Abednego

martinussatiapurwadi.abednego@unifr.ch

1 Earth Sciences, Department of Geosciences, University of Fribourg, Chemin du Musée 6, 1700 Fribourg, Switzerland

2 Institut für Geophysik, Universität Stuttgart, Azenbergstr. 16, 70174 Stuttgart, Germany

3 Federal Office of Topography swisstopo, Seftigenstrasse 264, 3084 Wabern, Switzerland seismotectonic interpretation. The synthetic focal mechanism can be linked to the main regional structural features: the NNE-SSW-oriented reactivated faults associated with the Rhine Graben development, and the NE-SW-oriented reverse faults related to the thrust development of major folds such as the Mont Terri anticline. The results for this pilot study confirm that our affirmative method can be used to augment local and regional seismotectonic interpretations with very weak-intensity earthquake data.

Keywords Underground and surface seismic mini-arrays . Microseismic activity - Focal mechanisms - Fault data . Synthetic P-phase waveform - Rhine Graben development

\section{Introduction}

The objective of this pilot study was to gain a better understanding of the seismotectonics and the link between different tectonic structures and seismicity of the Mont Terri region. We explored the potential of generating synthetic earthquake waveforms based on discrete observed fault families and using these to constrain existing but poorly defined microseismic earthquake data. These waveforms can be further utilised to determine focal mechanisms and hypocentre locations. We can then use these parameters to synthesize focal mechanisms for weakintensity seismic events by correlating recorded and synthetic waveform data from synthetic focal mechanisms.

The Mont Terri rock laboratory just north of St-Ursanne in the canton of Jura, Switzerland (Fig. 1) is a geoscience research facility located in the northernmost part of the Jura fold- and-thrust belt and dedicated to research on the hydrogeological, geochemical, and geotechnical properties focused on characterising the Opalinus Clay Formation. 
(a)

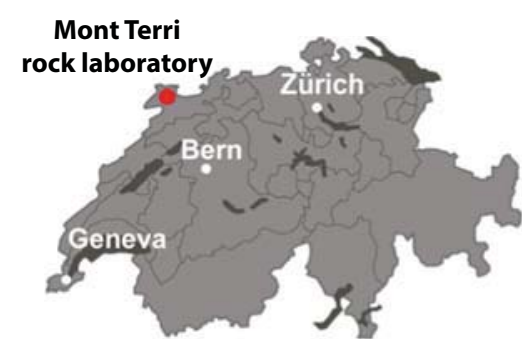

(c)

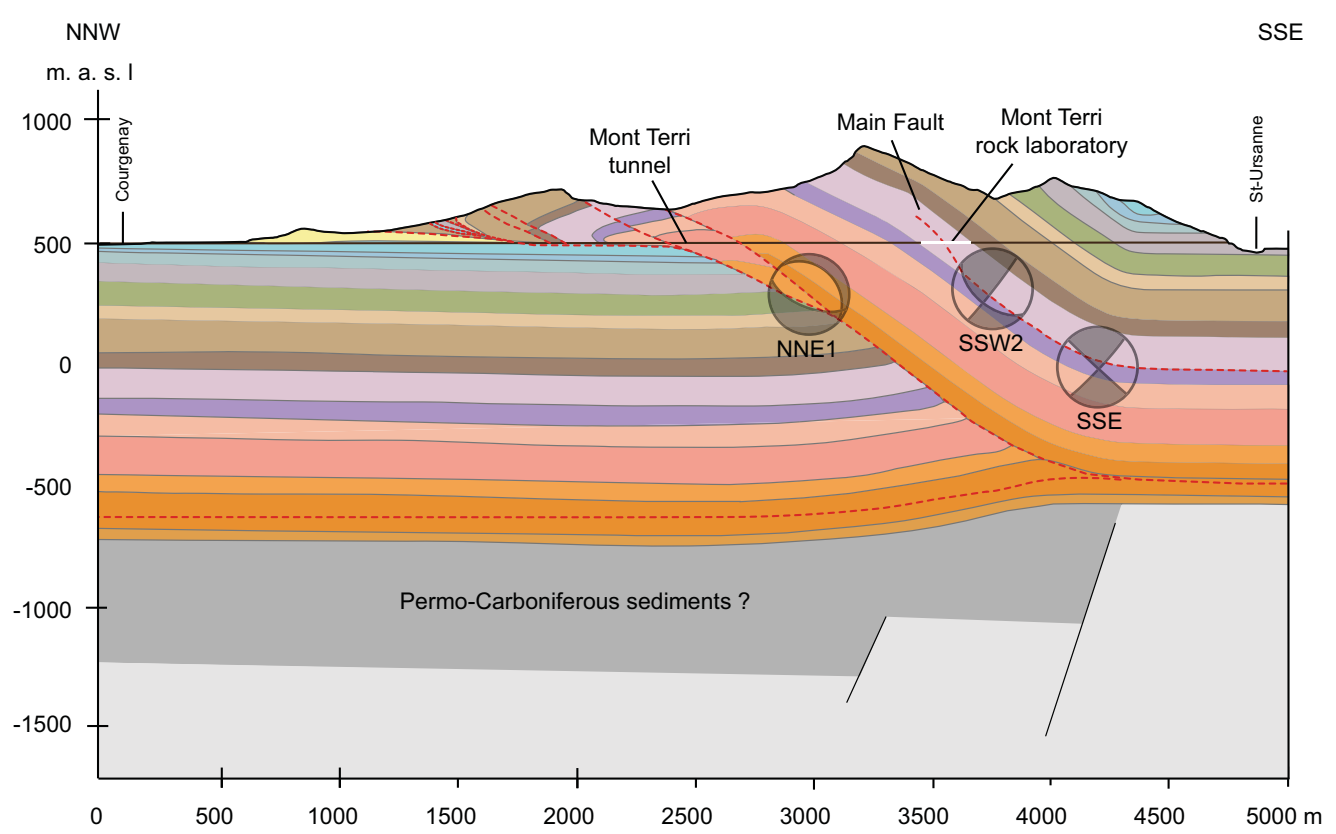

(b)
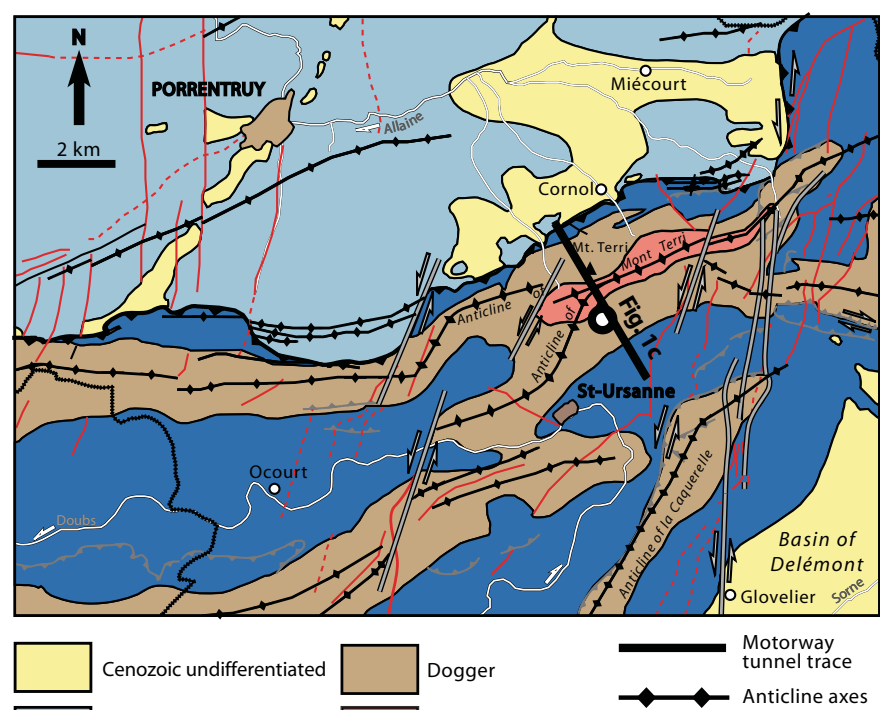

Lias and Keuper
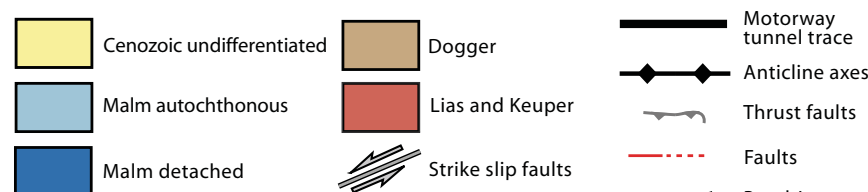

Strike slip faults

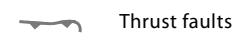

—...- Faults $\sim_{\substack{\text { Basal Jura } \\ \text { thrust }}}$ SSE
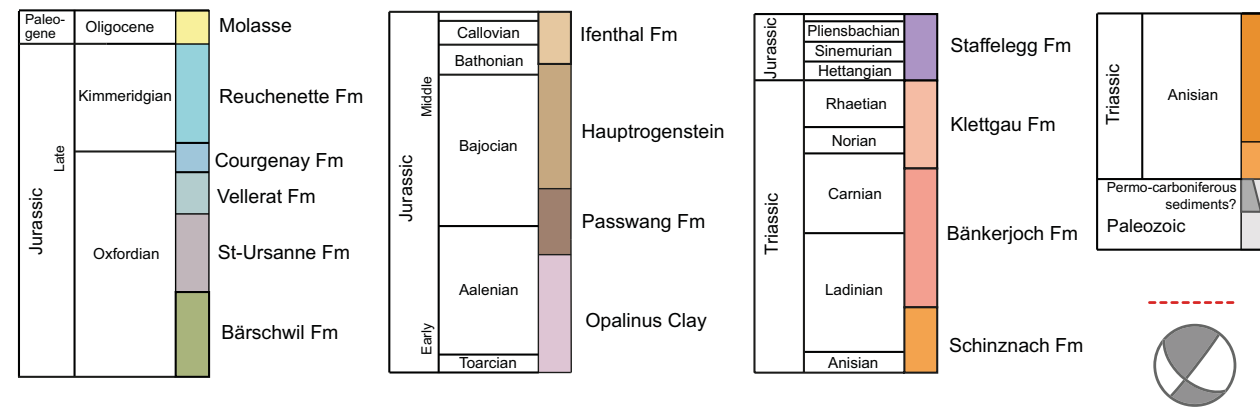

Zeglingen Fm

Kaiseraugst $\mathrm{Fm}$

basement basement
undifferentiated

Thrust planes

Focal mechanism (Back-section hemisphere projection) 
4Fig. 1 a Location of Mont Terri rock laboratory in the northwest of Switzerland. b Tectonic map indicating the rock laboratory situated in the Jura fold-and-thrust belt. The dominant structures are NNE-SSWtrending faults with NE-SW and EW fold axes. c Cross-section of the Mont Terri anticline (modified after Nussbaum et al. 2017) with backsection hemisphere projection of focal mechanisms of microseismic events in the vicinity of the Mont Terri rock laboratory corresponding to the sub-groups in Fig. 4

This clay formation is being considered as potential host rock for the deep geological repository of radioactive waste (NEA 1999; Thury and Bossart 1999). Structurally, Mont Terri corresponds to the Early Jurassic part of the detached, thrusted, and folded Mesozoic Jura series. The structural background to the underground laboratory is a large noncylindrical NE-NW-oriented ramp-related anticline and a major thrust fault, the Main Fault.

To better understand the seismic hazard in the Mont Terri area and its impact on a possible repository, we implemented a microseismic monitoring project. Instrumental monitoring of seismicity by the Swiss Seismological Service (SED) began in 1975 through the Swiss Digital Seismic Network (SDSNet) and the Swiss Strong Motion Network (SSMNet) (Cauzzi and Clinton 2013). Up to the end of 2008, the SED catalogued 133 events in the vicinity (radius of $20 \mathrm{~km}$ ) of the Mont Terri rock laboratory in their Earthquake Catalog of Switzerland (ECOS-09) (Fäh et al. 2011) (Fig. 2).

The data in this study are from four permanent SED stations in addition to a newly implemented local network (Seismic Navigation System-SNS). The SED stations are either very close to or within the rock laboratory itself (Fig. 3): two stations in galleries recording since May 19, 2014: the accelerometer MTI01 and the velocimeter MTI02; and two stations at the surface: the accelerometer MTI03 recording since October 1, 2014 and the velocimeter BOURR recording since April 26, 2000. In April 2014, two Seismic Navigation Systems (SNS), operating as seismic mini-arrays (Joswig 2008), were installed at the Mont Terri rock laboratory to increase the density of the existing seismic network in order to optimize monitoring of seismic activity in the vicinity. One station is located inside the rock laboratory and the other near the main south entrance to the safety gallery of the motorway tunnel. Additional SNS were installed at the surface in July 2015 at the locality of Monnat, and in September 2015 near La Combe. A temporary SNS was installed between May and November 2015 close to the Main Thrust within the rock laboratory to monitor any in situ fault slip during repeated hydraulic stimulation testing (FS Experiment, Guglielmi et al. 2016) (Fig. 3).

For this pilot study we considered the two-year monitoring-period between April 1, 2014 and December 31, 2015. During this time, the SED detected and located a total of 94 events within a radius of $30 \mathrm{~km}$ around the rock laboratory, of which 20 were located within a radius of $10 \mathrm{~km}$ of the rock laboratory (Table 1).

Microseismic event detection was performed with the NanoSeismicSuite. This software uses an auto-adaptive frequency dependence and noise-muting spectrogram called the sonogram of the seismic records (Joswig 2008; Sick et al. 2012) that are applied to the data sets from SED and SNS. Hypocentre location was done with HypoLine, an analyst-guided location software, combining network and array techniques (Joswig 2008; Vouillamoz et al. 2016) and additional constraints from records of permanent SED stations. The SNS monitoring has been running continuously since April 2014. Due to initial testing performed for the SNS with GPS calibrations, a consistent catalogue exists only as of July 2014. In all, 198 microseismic events were catalogued near the rock laboratory over the period July 2014 to August 2015, of which 86 events are within a radius of $10 \mathrm{~km}$ around the laboratory (Table 2; Fig. 3). Of these events, 10 were also catalogued by the SED (Table 1). The 198 events have local magnitudes ranging between $\mathrm{ML}=-2.0$ and $\mathrm{ML}=2.0$. These magnitudes are calculated based on horizontal maximum peak-to-peak amplitude readings of the stations and epicentral attenuation functions applied in HypoLine to the datasets from the SED and SNS network (Vouillamoz et al. 2016). Since these events have low local magnitude that were detected by only a few stations, we did not perform magnitude calibration for this specific area. However, using the entire magnitude range (EMR) method (Woessner and Wiemer 2005), we calculated the magnitude of completeness (Mc) to be $\mathrm{Mc}=0.5$, based on local magnitudes in a $20 \mathrm{~km}$ area around the Mont Terri rock laboratory.

\section{Focal mechanism analysis}

We used focal mechanism analysis to determine the orientation, slip direction, and sense of slip of the calculated nodal planes as defined by strike, dip, and rake (Aki and Richards 1980) and implicitly, the fault plane generating the seismicity. Focal mechanisms were calculated based on first-motion or waveform observation of seismograms recorded by the seismic stations. Using these two datasets there are two approaches for determining the focal mechanism of an earthquake: the unsupervised grid search approach, and the supervised synthetic waveform-correlation approach (Oppenheimer et al. 1988; Kobayashi and Nakanishi 1994; Zoback and Harjes 1997; Hardebeck and Shearer 2002).

In first-motion observations, signals generated by a seismic source have to be clearly recorded with a low noise level at the recording stations in order to determine polarity of the first arrival of the P-phase. A high number of 


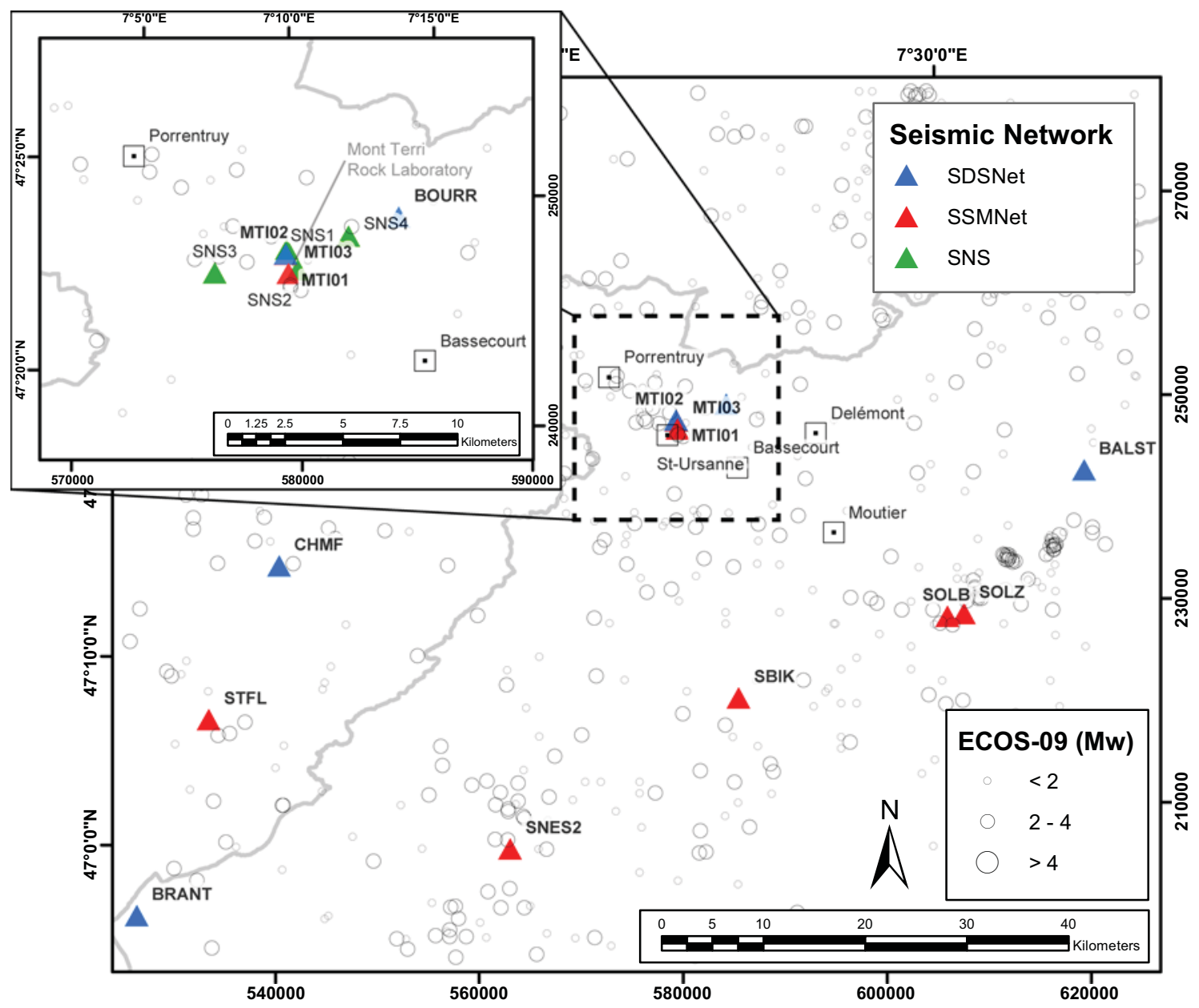

Fig. 2 Instrumentally recorded seismicity catalogued by the Swiss Seismological Service (SED) in the Earthquake Catalog of Switzerland (ECOS-09 (Fäh et al. 2011) from 1975 until 2008 in the vicinity of the Mont Terri rock laboratory. The SED monitors this area using the Digital Seismic Network (SDSNet) and the Swiss Strong Motion

recording stations and a spatial distribution with a good radial coverage will considerably improve constraining the focal mechanism solution using the unsupervised grid search approach. This condition is commonly found for seismic events with magnitude (ML) $>1.5-2.0$ (Plenefisch and Bonjer 1997).

Since the spatial distribution of the stations around the rock laboratory in our pilot study was irregular and the magnitudes (ML) of the events in the vicinity of the Mont Terri rock laboratory were below 2.0, the grid-search approach yields unconstrained focal mechanism solutions. Therefore, we decided to apply the supervised approach on waveform observations using synthetic waveform correlation to determine the focal mechanisms.

If we assume that an earthquake is generated on a given fault plane with known slip direction and sense then we can determine the two possible nodal planes (one being the active seismic fault plane) of the focal mechanism. This
Network (SSMNet) (Cauzzi and Clinton 2013). Seismic Navigation Systems (SNS) are mini-arrays installed in the vicinity of the Mont Terri rock laboratory since April 2014 for microseismic monitoring. The map is in the Swiss coordinate system CH1903

approach can also be applied to inherited faults for which the slip direction, and hence the paleostress, are known and could be reactivated in the present regional stress field.

\section{Synthetic focal mechanisms}

\subsection{In-situ measurements of faults in the Mesozoic cover}

Fault planes and striations were systematically measured and documented during the excavation of the galleries in the Mont Terri rock laboratory (Nussbaum et al. 2011). These were re-analyzed here in order to discriminate fault families and paleostress direction using the Stereonet and FaultKin software packages (Allmendinger et al. 1989a, b, 2012). For this re-analysis we used the "righthand-rule" convention to record orientations of 


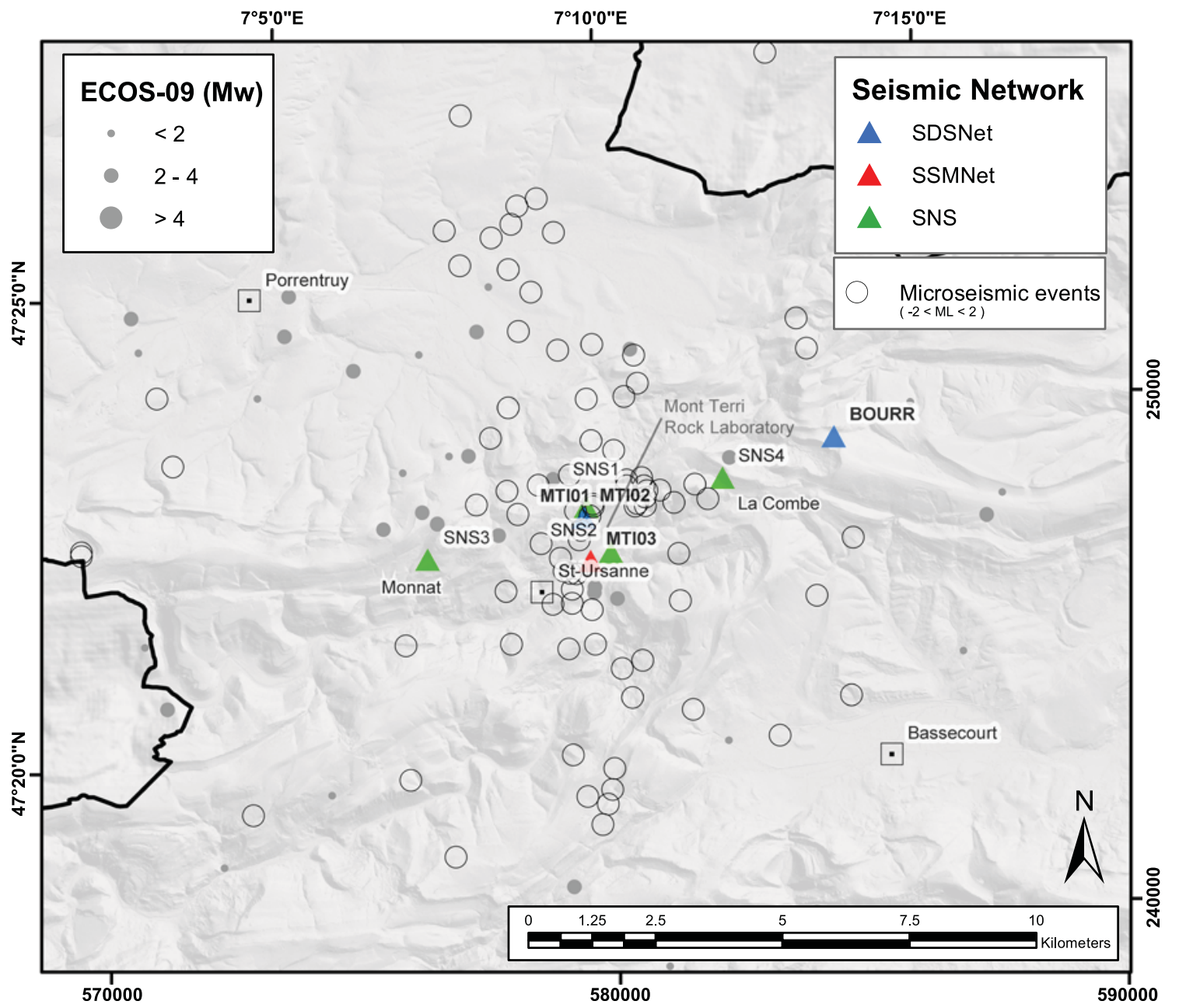

Fig. 3 Map of the Digital Seismic Network (SDSNet) and the Swiss Strong Motion Network (SSMNet) monitoring the seismicity and the Seismic Navigation System (SNS) mini-arrays monitoring the microseismicity in the vicinity of the Mont Terri rock laboratory.

slickenlines (or slip direction) and surfaces on which they occur. The slickenline rake is the angle measured clockwise between the strike direction and the slickenline on the surface of interest. We discriminated several groups of faults (Fig. 4). In total, 234 fault-striation pairs, mostly reverse faults (Nussbaum et al. 2011), were available to calculate the paleostress axes using the kinematic pressuretension (PT-axes) method (Marrett and Allmendinger 1990). The resulting main stress axis has $\mathrm{P}$-axis $337^{\circ} / 06^{\circ}$, T-axis $163^{\circ} / 84^{\circ}$, and $\mathrm{B}$ (intermediate)-axis $067^{\circ} / 01^{\circ}$. Taken together, all the axes show a rather consistent distribution with maximum horizontal compression $\left(\mathrm{SH}_{\max }\right)$ oriented NNW $337^{\circ} \pm 28^{\circ}$ (Fig. 5). We have used this mean paleostress axis to represent the present regional stress field. This choice is supported by the lack of any clear and reliable regional present-day stress indicator in the sedimentary cover. All measurements of paleostress in the cover have more or less the same orientation as the

The map shows 86 detected and located microseismic events around the Mont Terri rock laboratory catalogued between July 2014 and August 2015. The map is in the Swiss coordinate system CH1903

derived horizontal paleostress $\left(\mathrm{SH}_{\max } \mathrm{NNW}\right)$. Only one in situ measurement is known from the literature (Heidbach and Reinecker 2013) and it has almost the same orientation as the general one that we proposed here. Therefore we applied this mean stress field ( $\left.\mathrm{SH}_{\max } \mathrm{NNW}\right)$ to all subsequent parametric analyses of groups and sub-groups of faults (in the cover and basement) to generate synthetic focal mechanisms of earthquakes.

This stress inversion approach on fault/striation pairs can be further refined by sorting the data into 4 main groups based on direction and fault dip (Fig. 4): (1) NNEtrending, (2) SSE-dipping, (3) SSW-dipping, and (4) subhorizontal. Each group can be further subdivided into subgroups based on orientation of the slip lineation (Fig. 4). Each group can be represented by the simplified fault plane/auxiliary plane projection (equivalent to the nodal plane solution). It is thus possible to determine an ideal stress orientation for each respective group: (1) NNE- 
Table 1 List of 94 events detected by the Swiss Seismological Service (SED) within a radius of $30 \mathrm{~km}$ around the Mont Terri rock laboratory during the monitoring period between April 1, 2014 and December 31, 2015

\begin{tabular}{|c|c|c|c|c|c|c|}
\hline Time & Lat & Lon & $\begin{array}{l}\text { Depth } \\
(\mathrm{km})\end{array}$ & ML & Location & \\
\hline 2014/04/01 05:29:20 & 47.53 & 6.99 & 7.8 & - & Boncourt JU & \\
\hline 2014/04/03 09:00:00 & 47.36 & 7.37 & -0.4 & 1.2 & DELEMONT JU & \\
\hline 2014/04/05 04:25:58 & 47.48 & 7.42 & 18.7 & 1.2 & Laufen BL & \\
\hline 2014/04/07 10:08:16 & 47.23 & 7.38 & -1.0 & 1.3 & Grenchen SO & \\
\hline 2014/04/08 13:28:46 & 47.28 & 7.06 & -0.9 & 1.4 & Saignelegier JU & \\
\hline 2014/04/11 11:18:09 & 47.33 & 7.17 & 0.4 & 1.2 & St-Ursanne JU & $*$ \\
\hline 2014/04/22 11:02:21 & 47.23 & 7.15 & 8.1 & 1.7 & Tavannes BE & \\
\hline 2014/04/24 11:18:51 & 47.42 & 7.18 & 2.7 & - & St-Ursanne JU & $*$ \\
\hline 2014/05/12 13:13:07 & 47.19 & 7.44 & -0.4 & 1.4 & Grenchen SO & \\
\hline 2014/05/16 04:21:39 & 47.47 & 7.19 & 10.4 & 2.1 & Porrentruy JU & \\
\hline 2014/05/21 02:10:58 & 47.16 & 7.16 & 7.0 & 1.7 & Biel BE & \\
\hline 2014/05/28 19:43:21 & 47.15 & 7.17 & 6.6 & 1.0 & Biel BE & \\
\hline 2014/05/31 14:24:14 & 47.40 & 7.25 & 17.5 & 1.5 & St-Ursanne JU & $*$ \\
\hline 2014/05/31 15:44:55 & 47.40 & 7.26 & 17.3 & 0.9 & DELEMONT JU & $*$ \\
\hline 2014/06/03 13:25:04 & 47.28 & 7.14 & -0.8 & 1.3 & Tavannes BE & \\
\hline 2014/06/27 09:33:15 & 47.29 & 7.14 & 0.0 & 1.8 & St-Ursanne JU & $*$ \\
\hline 2014/07/01 15:49:08 & 47.23 & 7.25 & 2.6 & 1.6 & Tavannes BE & \\
\hline 2014/07/02 07:28:42 & 47.22 & 7.16 & 3.2 & 1.4 & Tavannes BE & \\
\hline 2014/07/03 10:24:56 & 47.65 & 7.28 & 1.0 & 1.5 & Mulhouse F & \\
\hline 2014/07/04 09:55:48 & 47.63 & 7.50 & 24.3 & - & BASEL & \\
\hline 2014/07/08 13:36:28 & 47.42 & 7.14 & 2.8 & - & Porrentruy JU & $*$ \\
\hline 2014/08/12 08:01:10 & 47.33 & 7.17 & 2.6 & 1.3 & St-Ursanne JU & $*$ \\
\hline 2014/08/19 03:44:50 & 47.29 & 7.56 & 4.7 & 1.1 & Welschenrohr SO & \\
\hline 2014/08/19 03:47:23 & 47.28 & 7.56 & 6.4 & 1.8 & Welschenrohr SO & \\
\hline 2014/08/22 05:25:59 & 47.16 & 7.14 & 10.9 & 1.6 & Tavannes BE & \\
\hline 2014/08/24 01:39:07 & 47.29 & 7.56 & 5.5 & 1.2 & Welschenrohr SO & \\
\hline 2014/08/26 07:35:12 & 47.25 & 7.21 & -1.0 & 1.4 & Tavannes BE & \\
\hline 2014/09/11 00:12:29 & 47.15 & 7.16 & 10.2 & 1.4 & Biel BE & \\
\hline 2014/09/30 17:27:15 & 47.32 & 7.20 & 17.9 & 1.1 & St-Ursanne JU & $*$ \\
\hline 2014/10/06 07:32:54 & 47.24 & 7.21 & -0.7 & 1.4 & Tavannes BE & \\
\hline 2014/10/28 15:16:37 & 47.33 & 7.20 & -0.4 & 1.4 & St-Ursanne JU & $*$ \\
\hline 2014/11/14 13:40:48 & 47.32 & 7.18 & -0.7 & 1.2 & St-Ursanne JU & $*$ \\
\hline 2014/11/19 08:32:36 & 47.20 & 7.25 & 31.7 & 1.3 & Tavannes BE & \\
\hline 2014/11/22 13:03:31 & 47.19 & 7.05 & 0.0 & - & St-Imier BE & \\
\hline 2014/12/30 08:29:25 & 47.22 & 7.14 & 10.2 & 1.1 & Tavannes BE & \\
\hline 2015/01/06 23:31:21 & 47.33 & 7.09 & 21.5 & 1.2 & St-Ursanne JU & $*$ \\
\hline 2015/01/10 08:12:24 & 47.28 & 7.24 & 18.2 & 1.0 & Tavannes BE & \\
\hline 2015/01/31 21:23:29 & 47.15 & 7.14 & 10.3 & 1.6 & Biel BE & \\
\hline 2015/01/31 21:54:02 & 47.15 & 7.15 & 9.7 & 3.1 & Biel BE & \\
\hline 2015/01/31 22:20:56 & 47.15 & 7.14 & 10.9 & 2.1 & Biel BE & \\
\hline 2015/02/01 00:20:57 & 47.15 & 7.14 & 10.5 & 1.1 & Switzerland & \\
\hline 2015/02/01 12:26:39 & 47.15 & 7.14 & 9.2 & 1.6 & Biel BE & \\
\hline 2015/02/01 16:50:31 & 47.23 & 7.10 & 20.4 & 1.2 & Tavannes BE & \\
\hline 2015/02/01 18:04:06 & 47.19 & 7.00 & 5.0 & - & St-Imier BE & \\
\hline 2015/02/01 19:57:51 & 47.23 & 7.11 & 23.4 & 1.0 & Tavannes BE & \\
\hline 2015/02/02 04:05:18 & 47.15 & 7.15 & 10.1 & 2.6 & Biel BE & \\
\hline 2015/02/05 13:42:39 & 47.23 & 7.10 & 20.1 & 0.8 & Tavannes BE & \\
\hline 2015/02/05 15:42:23 & 47.17 & 7.12 & 11.6 & 0.7 & Tavannes BE & \\
\hline
\end{tabular}


Table 1 continued

\begin{tabular}{|c|c|c|c|c|c|c|}
\hline Time & Lat & Lon & $\begin{array}{l}\text { Depth } \\
(\mathrm{km})\end{array}$ & ML & Location & \\
\hline 2015/02/20 12:33:11 & 47.43 & 7.02 & -0.2 & 1.7 & Porrentruy JU & \\
\hline 2015/02/23 00:52:09 & 47.15 & 7.14 & 9.9 & 1.1 & Biel BE & \\
\hline 2015/03/14 19:35:55 & 47.35 & 6.85 & 4.9 & - & Saignelegier JU & \\
\hline 2015/03/15 08:21:38 & 47.18 & 7.11 & 7.0 & 1.0 & Tavannes BE & \\
\hline 2015/03/18 15:03:28 & 47.37 & 7.16 & 7.0 & 1.5 & St-Ursanne JU & $*$ \\
\hline 2015/03/31 12:34:33 & 47.23 & 7.38 & 6.9 & - & Grenchen SO & \\
\hline 2015/04/09 00:05:01 & 47.15 & 7.14 & 10.6 & 1.3 & Biel BE & \\
\hline 2015/04/14 11:02:10 & 47.20 & 7.24 & -1.0 & 1.6 & Tavannes BE & \\
\hline 2015/04/18 23:16:25 & 47.57 & 7.33 & 7.0 & 2.0 & Mulhouse F & \\
\hline 2015/04/22 11:40:53 & 47.39 & 7.03 & -0.2 & - & Porrentruy JU & \\
\hline 2015/04/28 12:16:28 & 47.37 & 7.12 & 10.3 & 1.5 & St-Ursanne JU & $*$ \\
\hline 2015/04/30 02:39:50 & 47.65 & 7.42 & 14.8 & 1.4 & Mulhouse F & \\
\hline 2015/05/09 18:34:52 & 47.15 & 7.15 & 9.8 & 2.4 & Biel BE & \\
\hline 2015/05/09 22:18:29 & 47.15 & 7.16 & 9.5 & 1.2 & Biel BE & \\
\hline 2015/05/10 03:03:49 & 47.15 & 7.15 & 10.4 & 1.2 & Biel BE & \\
\hline 2015/05/14 16:28:18 & 47.15 & 7.17 & 6.0 & 1.0 & Biel BE & \\
\hline 2015/05/20 12:19:19 & 47.33 & 7.20 & -0.4 & - & St-Ursanne JU & $*$ \\
\hline 2015/05/22 13:31:29 & 47.64 & 6.93 & 3.1 & 2.3 & Belfort F & \\
\hline 2015/06/08 21:47:10 & 47.34 & 6.93 & 4.1 & 1.4 & Saignelegier JU & \\
\hline 2015/06/12 09:48:33 & 47.38 & 7.19 & -0.7 & 1.3 & St-Ursanne JU & $*$ \\
\hline 2015/06/28 13:27:22 & 47.59 & 7.42 & 14.4 & 0.9 & BASEL & \\
\hline 2015/06/28 13:38:13 & 47.58 & 7.46 & 14.8 & 0.4 & BASEL & \\
\hline 2015/07/08 11:17:38 & 47.20 & 7.20 & -1.0 & 1.7 & Tavannes BE & \\
\hline 2015/07/21 07:27:16 & 47.17 & 7.17 & -1.2 & 1.4 & Tavannes BE & \\
\hline 2015/07/22 07:18:56 & 47.19 & 7.24 & -0.6 & 1.6 & Tavannes BE & \\
\hline 2015/08/03 07:30:35 & 47.18 & 7.21 & 3.9 & 1.3 & Tavannes BE & \\
\hline 2015/08/07 05:12:13 & 47.36 & 7.05 & 6.2 & 1.7 & Porrentruy JU & $*$ \\
\hline 2015/08/09 12:31:21 & 47.36 & 7.05 & 6.1 & 1.6 & Porrentruy JU & $*$ \\
\hline 2015/08/14 11:33:50 & 47.27 & 7.15 & -0.7 & 1.0 & Tavannes BE & \\
\hline 2015/08/17 23:31:45 & 47.36 & 7.05 & 3.7 & 0.8 & Porrentruy JU & $*$ \\
\hline 2015/08/18 13:40:27 & 47.34 & 7.20 & 0.0 & - & St-Ursanne JU & * \\
\hline 2015/09/09 03:01:49 & 47.28 & 7.28 & 11.4 & 1.0 & Moutier BE & \\
\hline 2015/09/18 12:30:47 & 47.32 & 7.18 & -0.8 & 1.2 & St-Ursanne JU & * \\
\hline 2015/09/20 21:48:37 & 47.45 & 7.49 & 9.6 & 0.5 & Laufen BL & \\
\hline 2015/09/20 21:50:31 & 47.45 & 7.49 & 8.5 & 0.5 & Laufen BL & \\
\hline 2015/10/02 07:24:02 & 47.24 & 7.19 & -0.8 & 1.4 & Tavannes BE & \\
\hline 2015/10/21 10:56:44 & 47.18 & 7.26 & -0.8 & 1.1 & Biel BE & \\
\hline 2015/10/26 08:31:43 & 47.22 & 7.21 & -0.8 & 1.6 & Tavannes BE & \\
\hline 2015/11/06 08:28:22 & 47.20 & 7.16 & -0.7 & 1.4 & Tavannes BE & \\
\hline 2015/11/10 15:01:31 & 47.17 & 7.22 & -1.2 & 1.7 & Biel BE & \\
\hline 2015/11/16 02:08:23 & 47.61 & 7.28 & 8.0 & 1.8 & Mulhouse F & \\
\hline 2015/11/27 08:33:18 & 47.20 & 7.20 & 15.2 & 1.5 & Tavannes BE & \\
\hline 2015/12/03 14:01:06 & 47.53 & 7.06 & -0.1 & - & Boncourt JU & \\
\hline 2015/12/08 22:34:32 & 47.63 & 7.30 & 8.2 & 0.7 & Mulhouse F & \\
\hline 2015/12/10 08:33:46 & 47.18 & 7.19 & -0.9 & 1.5 & Tavannes BE & \\
\hline 2015/12/21 12:22:22 & 47.20 & 7.35 & 17.1 & 1.4 & Grenchen SO & \\
\hline
\end{tabular}

The rows marked with a star show the 20 events within a radius of $10 \mathrm{~km}$ around the laboratory 
Table 2 List of 198 detected and located microseismic events around the Mont Terri rock laboratory monitored in this study from July 2014 until August 2015 using NanoSeismicSuite and HypoLine software (Joswig 2008; Sick et al. 2012; Vouillamoz et al. 2016)

\begin{tabular}{|c|c|c|c|c|c|}
\hline Time & Lat & Lon & Depth (km) & ML & \\
\hline 2014/07/02 01:12:33 & 46.78 & 6.07 & 6.3 & 0.6 & \\
\hline 2014/07/02 07:28:43 & 47.24 & 7.14 & 7.3 & 0.7 & \\
\hline 2014/07/03 10:24:57 & 47.63 & 7.28 & 7.3 & 0.8 & \\
\hline 2014/07/03 15:26:10 & 47.57 & 8.32 & 6.1 & - & \\
\hline 2014/07/04 19:36:13 & 47.26 & 7.06 & 12.3 & 0.0 & \\
\hline 2014/07/04 19:42:58 & 47.34 & 7.22 & 4.5 & - & $*$ \\
\hline 2014/07/08 13:36:28 & 47.40 & 7.18 & 1.3 & 0.8 & $* *$ \\
\hline 2014/07/08 13:36:28 & 47.40 & 7.15 & 1.5 & 0.9 & $*$ \\
\hline 2014/07/09 07:32:05 & 47.17 & 7.22 & 9.8 & 0.9 & \\
\hline 2014/07/09 10:58:35 & 48.05 & 7.80 & 4.4 & - & \\
\hline 2014/07/09 12:56:01 & 47.07 & 8.22 & 6.3 & - & \\
\hline 2014/07/10 09:56:10 & 47.52 & 6.64 & 9.7 & 1.5 & \\
\hline 2014/07/14 04:40:54 & 47.99 & 7.04 & 7.4 & 0.8 & \\
\hline 2014/07/14 10:42:39 & 47.04 & 7.63 & 9.0 & 0.6 & \\
\hline 2014/07/16 16:12:15 & 48.49 & 6.92 & 8.9 & 1.4 & \\
\hline 2014/07/17 15:08:51 & 47.03 & 6.86 & 8.6 & - & \\
\hline 2014/07/18 09:33:08 & 48.11 & 6.58 & 9.0 & 1.6 & \\
\hline 2014/07/18 10:02:14 & 48.40 & 6.84 & 9.1 & 1.5 & \\
\hline 2014/07/22 10:13:32 & 48.48 & 6.82 & 8.9 & 1.6 & \\
\hline 2014/07/24 08:55:53 & 47.56 & 7.33 & 9.0 & 1.7 & \\
\hline 2014/07/24 08:55:55 & 47.45 & 7.16 & 8.5 & 1.2 & $*$ \\
\hline 2014/07/24 10:17:14 & 48.53 & 6.76 & 8.9 & 1.4 & \\
\hline 2014/07/24 14:10:22 & 47.24 & 8.24 & 9.0 & - & \\
\hline 2014/07/25 10:15:16 & 48.51 & 6.81 & 8.9 & 1.5 & \\
\hline $2014 / 07 / 25 \quad 15: 35: 23$ & 47.48 & 7.17 & 6.8 & -0.3 & \\
\hline 2014/07/27 04:37:16 & 47.31 & 7.23 & 3.0 & - & $*$ \\
\hline 2014/08/01 09:56:00 & 48.38 & 6.82 & 11.1 & 1.3 & \\
\hline 2014/08/01 10:03:44 & 47.69 & 7.29 & 10.2 & 0.9 & \\
\hline 2014/08/01 14:39:55 & 46.57 & 6.78 & 11.6 & 0.9 & \\
\hline 2014/08/01 16:10:04 & 46.61 & 6.82 & 7.5 & - & \\
\hline 2014/08/03 18:26:02 & 47.36 & 7.14 & -0.1 & -1.3 & $*$ \\
\hline 2014/08/04 10:02:14 & 48.41 & 6.85 & 17.7 & 1.5 & \\
\hline 2014/08/06 14:23:46 & 47.69 & 5.80 & 8.6 & 1.3 & \\
\hline 2014/08/07 09:11:18 & 46.49 & 7.36 & 10.6 & - & \\
\hline 2014/08/08 08:59:35 & 47.17 & 8.16 & 8.1 & - & \\
\hline 2014/08/12 08:01:10 & 47.32 & 7.17 & 2.1 & - & $* *$ \\
\hline 2014/08/12 09:07:22 & 47.80 & 6.85 & 6.5 & 1.6 & \\
\hline 2014/08/14 13:44:13 & 47.08 & 7.36 & -0.3 & - & \\
\hline 2014/08/15 00:44:56 & 47.25 & 7.58 & 7.9 & 0.2 & \\
\hline 2014/08/15 08:59:26 & 47.11 & 8.16 & 10.9 & - & \\
\hline 2014/08/16 11:40:20 & 47.94 & 7.23 & 7.4 & 0.7 & \\
\hline 2014/08/19 10:06:43 & 48.38 & 6.84 & 9.1 & 1.2 & \\
\hline 2014/08/21 10:18:59 & 48.50 & 6.76 & 8.9 & 1.6 & \\
\hline 2014/08/22 12:07:03 & 47.09 & 6.92 & 9.1 & 1.4 & \\
\hline 2014/08/26 08:05:28 & 47.37 & 7.35 & 0.3 & 1.3 & \\
\hline 2014/08/26 08:14:55 & 48.45 & 6.67 & 9.0 & 1.6 & \\
\hline
\end{tabular}

Table 2 continued

\begin{tabular}{|c|c|c|c|c|c|}
\hline Time & Lat & Lon & Depth $(\mathrm{km})$ & ML & \\
\hline 2014/08/26 08:15:56 & 47.38 & 7.19 & -0.3 & -1.5 & $*$ \\
\hline 2014/08/26 18:46:12 & 47.49 & 7.53 & 11.6 & 0.3 & \\
\hline 2014/08/29 11:41:38 & 47.24 & 7.10 & 8.5 & 0.6 & \\
\hline 2014/09/05 10:12:00 & 48.39 & 6.72 & 19.6 & 1.7 & \\
\hline 2014/09/09 11:30:16 & 47.38 & 7.18 & 2.3 & -0.9 & $*$ \\
\hline 2014/09/09 13:44:02 & 47.25 & 7.47 & 6.2 & - & \\
\hline 2014/09/11 13:21:11 & 47.26 & 7.11 & 17.2 & 0.9 & \\
\hline 2014/09/15 08:24:46 & 47.25 & 7.16 & 6.0 & - & \\
\hline 2014/09/19 08:32:13 & 47.58 & 7.32 & 8.4 & 1.7 & \\
\hline 2014/09/23 09:00:27 & 47.55 & 6.88 & 6.0 & 1.5 & \\
\hline 2014/09/25 10:04:38 & 48.54 & 6.83 & 8.9 & 1.8 & \\
\hline 2014/09/30 10:16:41 & 47.83 & 6.19 & 13.8 & - & \\
\hline 2014/09/30 17:27:15 & 47.28 & 7.20 & 14.6 & 0.7 & $* *$ \\
\hline 2014/10/01 09:31:29 & 47.51 & 7.41 & 10.4 & 1.7 & \\
\hline 2014/10/01 13:05:06 & 47.41 & 7.01 & 8.5 & - & \\
\hline 2014/10/02 10:21:58 & 47.38 & 7.14 & 0.4 & -1.1 & $*$ \\
\hline 2014/10/06 07:58:16 & 47.46 & 7.21 & 3.9 & 1.4 & $*$ \\
\hline 2014/10/09 15:05:56 & 48.14 & 6.43 & 24.5 & 1.4 & \\
\hline 2014/10/09 17:48:01 & 47.37 & 7.19 & -0.3 & - & $*$ \\
\hline 2014/10/10 04:31:46 & 47.41 & 6.26 & 14.6 & 0.9 & \\
\hline 2014/10/10 08:22:26 & 47.34 & 7.16 & 1.2 & 0.9 & $*$ \\
\hline 2014/10/11 21:36:43 & 47.36 & 7.17 & -0.3 & - & $*$ \\
\hline 2014/10/12 23:31:03 & 47.35 & 7.16 & 0.1 & -1.4 & $*$ \\
\hline 2014/10/13 07:30:31 & 47.17 & 7.23 & 7.9 & 1.0 & \\
\hline 2014/10/13 09:37:33 & 47.19 & 6.01 & 8.4 & 1.4 & \\
\hline 2014/10/13 13:59:29 & 47.18 & 7.24 & 9.7 & 0.9 & \\
\hline 2014/10/13 23:13:36 & 47.37 & 7.16 & 0.9 & -1.2 & $*$ \\
\hline 2014/10/17 07:12:41 & 47.11 & 7.15 & 10.7 & 1.4 & \\
\hline 2014/10/20 10:08:36 & 48.38 & 6.85 & 15.7 & 1.2 & \\
\hline 2014/10/22 01:46:22 & 47.35 & 7.23 & -0.3 & - & $*$ \\
\hline 2014/10/22 01:46:22 & 47.36 & 7.22 & 1.4 & -0.9 & $*$ \\
\hline 2014/10/23 09:03:28 & 47.69 & 6.88 & 3.9 & - & \\
\hline 2014/10/23 13:31:37 & 47.38 & 7.40 & 5.3 & 0.6 & \\
\hline 2014/10/27 15:23:03 & 46.21 & 7.20 & 9.4 & - & \\
\hline 2014/10/28 15:16:37 & 47.35 & 7.18 & 2.9 & - & $* *$ \\
\hline 2014/10/31 14:56:49 & 47.26 & 6.44 & 7.9 & 1.5 & \\
\hline 2014/10/31 22:31:12 & 47.41 & 7.22 & 7.0 & -0.8 & $*$ \\
\hline 2014/11/03 16:30:22 & 47.51 & 6.63 & 10.0 & 1.3 & \\
\hline 2014/11/05 16:53:31 & 48.41 & 6.86 & 10.9 & 1.5 & \\
\hline 2014/11/06 01:45:57 & 47.06 & 7.03 & 7.3 & - & \\
\hline 2014/11/07 11:59:41 & 47.12 & 8.15 & 7.7 & - & \\
\hline 2014/11/09 21:11:50 & 47.65 & 6.22 & 8.7 & - & \\
\hline 2014/11/13 08:44:16 & 47.31 & 7.24 & 7.7 & 0.3 & \\
\hline 2014/11/14 09:52:21 & 47.38 & 7.15 & 0.0 & -1.0 & $*$ \\
\hline 2014/11/14 10:29:34 & 47.38 & 7.14 & -0.3 & -0.9 & $*$ \\
\hline 2014/11/14 13:40:48 & 47.33 & 7.17 & -0.3 & - & $* *$ \\
\hline 2014/11/18 12:12:37 & 47.17 & 7.49 & 11.5 & 1.1 & \\
\hline 2014/11/21 14:34:45 & 47.45 & 6.74 & 6.3 & - & \\
\hline
\end{tabular}


Table 2 continued

\begin{tabular}{|c|c|c|c|c|c|}
\hline Time & Lat & Lon & Depth $(\mathrm{km})$ & ML & \\
\hline 2014/11/22 13:03:29 & 47.55 & 6.90 & 3.4 & 0.6 & \\
\hline 2014/11/25 14:40:32 & 47.39 & 6.96 & 9.2 & 1.2 & \\
\hline 2014/11/26 08:26:33 & 47.56 & 7.35 & 2.3 & 1.7 & \\
\hline 2014/12/01 11:12:09 & 47.25 & 7.01 & 10.7 & 0.2 & \\
\hline 2014/12/01 15:11:02 & 46.94 & 6.68 & -1.1 & - & \\
\hline 2014/12/02 11:48:27 & 47.15 & 7.12 & 6.2 & - & \\
\hline 2014/12/02 13:52:06 & 47.49 & 7.01 & 0.0 & 1.2 & \\
\hline 2014/12/05 11:40:09 & 47.41 & 7.17 & 1.6 & 0.0 & $*$ \\
\hline 2014/12/08 01:07:49 & 47.39 & 7.17 & -0.3 & -1.5 & $*$ \\
\hline 2014/12/09 07:51:59 & 48.14 & 6.51 & 23.7 & 1.4 & \\
\hline 2014/12/11 15:28:55 & 47.37 & 7.23 & -0.3 & -0.9 & $*$ \\
\hline 2014/12/11 18:17:24 & 46.75 & 6.93 & 17.8 & 1.0 & \\
\hline 2014/12/13 13:51:28 & 47.38 & 7.18 & -0.3 & -1.4 & $*$ \\
\hline 2014/12/13 14:18:16 & 47.38 & 7.18 & -0.3 & -1.3 & $*$ \\
\hline 2014/12/14 07:25:54 & 47.39 & 7.16 & -0.3 & -2.0 & $*$ \\
\hline 2014/12/14 17:59:06 & 47.37 & 7.16 & 0.0 & -1.8 & $*$ \\
\hline 2014/12/17 13:47:11 & 48.23 & 8.22 & 10.8 & 1.9 & \\
\hline $2014 / 12 / 20 \quad 13: 36: 58$ & 47.38 & 7.18 & -0.3 & -1.2 & * \\
\hline 2014/12/23 00:19:35 & 46.93 & 7.14 & 6.4 & 0.2 & \\
\hline 2014/12/24 15:03:03 & 47.17 & 7.60 & 5.1 & 0.2 & \\
\hline 2014/12/27 20:39:03 & 47.38 & 7.15 & -0.3 & -1.9 & $*$ \\
\hline 2015/01/04 01:29:04 & 47.40 & 7.17 & 2.9 & -1.4 & $*$ \\
\hline 2015/01/04 06:32:06 & 47.38 & 7.16 & 0.8 & -2.0 & $*$ \\
\hline 2015/01/06 09:48:52 & 47.51 & 7.36 & 16.8 & 1.8 & \\
\hline 2015/01/06 13:31:44 & 47.39 & 7.17 & 1.8 & -0.8 & * \\
\hline 2015/01/08 14:12:11 & 47.38 & 7.18 & 2.4 & - & * \\
\hline 2015/01/08 14:17:35 & 47.38 & 7.18 & 2.4 & -1.0 & $*$ \\
\hline 2015/01/13 09:30:46 & 47.43 & 7.15 & 0.4 & 0.5 & $*$ \\
\hline 2015/01/14 12:57:21 & 47.38 & 7.19 & -0.2 & -0.5 & $*$ \\
\hline 2015/01/17 11:35:45 & 47.54 & 7.21 & 5.0 & 0.3 & \\
\hline 2015/01/26 17:39:46 & 47.39 & 7.14 & 0.0 & -0.9 & $*$ \\
\hline 2015/01/27 00:58:02 & 47.40 & 7.16 & 5.5 & -0.6 & $*$ \\
\hline 2015/01/28 01:26:03 & 47.36 & 7.16 & 0.8 & -1.4 & $*$ \\
\hline 2015/02/01 04:02:48 & 47.18 & 7.11 & 15.4 & - & \\
\hline 2015/02/01 11:23:52 & 47.17 & 7.12 & 7.7 & 0.2 & \\
\hline 2015/02/01 18:04:05 & 47.13 & 7.15 & 6.4 & 0.6 & \\
\hline 2015/02/17 08:48:12 & 47.43 & 7.37 & 21.4 & 1.8 & \\
\hline 2015/02/22 13:58:28 & 47.15 & 7.13 & 4.0 & 0.4 & \\
\hline 2015/02/26 14:59:43 & 47.15 & 7.24 & 6.0 & 1.1 & \\
\hline 2015/03/05 10:13:36 & 47.41 & 7.22 & -0.3 & - & $*$ \\
\hline 2015/03/06 12:37:10 & 47.37 & 7.03 & 0.3 & 1.0 & $*$ \\
\hline 2015/03/12 10:29:22 & 47.37 & 7.03 & 0.3 & 0.7 & $*$ \\
\hline 2015/03/18 15:03:28 & 47.33 & 7.17 & 2.0 & 1.1 & $* *$ \\
\hline 2015/03/23 14:30:47 & 47.37 & 7.03 & 0.3 & 0.8 & \\
\hline 2015/03/24 12:12:19 & 47.43 & 7.15 & 2.0 & 1.2 & $*$ \\
\hline 2015/04/02 09:24:38 & 47.27 & 7.38 & 9.7 & 0.7 & \\
\hline 2015/04/04 04:11:28 & 47.37 & 7.15 & -0.3 & -1.8 & $*$ \\
\hline 2015/04/07 12:37:01 & 47.43 & 7.16 & 1.1 & 1.2 & * \\
\hline
\end{tabular}

Table 2 continued

\begin{tabular}{|c|c|c|c|c|c|}
\hline Time & Lat & Lon & Depth $(\mathrm{km})$ & ML & \\
\hline 2015/04/07 18:40:30 & 47.34 & 7.19 & -0.3 & - & $*$ \\
\hline 2015/04/08 14:22:16 & 47.42 & 7.14 & 0.9 & -0.1 & $*$ \\
\hline 2015/04/09 05:54:22 & 47.16 & 7.12 & 5.9 & 0.4 & \\
\hline 2015/04/13 12:02:21 & 47.40 & 7.05 & 2.5 & - & $*$ \\
\hline 2015/04/16 02:45:47 & 47.37 & 7.16 & -0.3 & - & $*$ \\
\hline 2015/04/16 09:27:40 & 47.40 & 7.14 & 4.2 & 0.0 & $*$ \\
\hline 2015/04/17 09:22:58 & 47.38 & 7.02 & 0.3 & 1.2 & \\
\hline 2015/04/17 12:58:06 & 47.36 & 7.15 & -0.3 & -0.9 & $*$ \\
\hline 2015/04/20 13:23:49 & 47.41 & 7.15 & 2.4 & 0.1 & $*$ \\
\hline 2015/04/22 07:12:02 & 47.37 & 7.16 & -0.3 & - & $*$ \\
\hline 2015/04/28 12:16:28 & 47.32 & 7.13 & 4.8 & 1.2 & $* *$ \\
\hline 2015/05/05 00:19:42 & 47.38 & 7.16 & -0.3 & - & $*$ \\
\hline 2015/05/05 09:47:06 & 47.52 & 7.40 & 2.0 & 1.7 & \\
\hline 2015/05/07 08:37:39 & 47.57 & 7.29 & 1.7 & 1.7 & \\
\hline 2015/05/08 09:48:27 & 47.45 & 6.95 & 3.6 & 1.7 & \\
\hline 2015/05/12 13:00:29 & 47.43 & 7.13 & 0.0 & 1.0 & $*$ \\
\hline 2015/05/15 07:30:27 & 47.24 & 7.16 & 13.6 & 0.8 & \\
\hline 2015/05/18 18:09:14 & 47.15 & 7.17 & 8.9 & 0.5 & \\
\hline 2015/05/20 12:19:18 & 47.33 & 7.17 & 1.5 & 0.9 & $* *$ \\
\hline $2015 / 05 / 22 \quad 13: 31: 33$ & 47.43 & 7.14 & 1.1 & 0.9 & $*$ \\
\hline 2015/05/27 09:28:41 & 47.42 & 7.13 & 3.3 & 1.1 & $*$ \\
\hline 2015/06/01 09:50:49 & 47.15 & 7.15 & 10.7 & 0.4 & \\
\hline 2015/06/01 10:58:35 & 47.55 & 6.89 & 6.2 & 1.5 & \\
\hline 2015/06/01 11:59:51 & 47.38 & 7.18 & -0.3 & 0.0 & $*$ \\
\hline 2015/06/01 21:26:52 & 47.16 & 7.12 & 5.9 & -0.2 & \\
\hline 2015/06/03 10:06:30 & 47.38 & 7.18 & -0.3 & 0.8 & $*$ \\
\hline 2015/06/05 09:36:59 & 47.56 & 7.32 & 3.5 & 1.7 & \\
\hline 2015/06/06 11:40:20 & 47.37 & 7.16 & 0.1 & 1.7 & $*$ \\
\hline 2015/06/08 21:47:09 & 47.36 & 6.92 & 6.5 & 1.1 & \\
\hline 2015/06/11 11:59:39 & 47.33 & 7.17 & 1.7 & 1.1 & $*$ \\
\hline 2015/06/12 09:48:32 & 47.38 & 7.18 & 0.2 & 0.9 & $* *$ \\
\hline 2015/06/19 10:01:12 & 47.38 & 7.18 & -0.3 & 0.5 & $*$ \\
\hline 2015/06/22 07:29:19 & 47.19 & 7.22 & 9.9 & 1.2 & \\
\hline 2015/06/27 13:37:08 & 47.45 & 7.13 & 0.8 & -0.6 & $*$ \\
\hline 2015/06/29 11:09:59 & 47.41 & 7.16 & 6.1 & 0.4 & $*$ \\
\hline 2015/06/30 12:23:30 & 47.42 & 7.15 & 0.8 & -0.2 & $*$ \\
\hline 2015/07/01 08:50:10 & 47.55 & 7.33 & 10.6 & 1.5 & \\
\hline 2015/07/07 05:05:06 & 47.38 & 7.20 & 0.8 & - & $*$ \\
\hline 2015/07/07 08:46:14 & 47.41 & 7.18 & 2.8 & -0.8 & $*$ \\
\hline 2015/07/08 08:06:19 & 47.06 & 7.17 & 10.5 & - & \\
\hline 2015/07/09 21:25:47 & 47.38 & 7.17 & -0.3 & - & $*$ \\
\hline 2015/07/14 12:54:04 & 47.33 & 7.12 & 2.8 & 0.0 & $*$ \\
\hline 2015/07/16 05:33:19 & 47.43 & 7.14 & 2.0 & 0.6 & $*$ \\
\hline 2015/07/18 07:14:14 & 47.36 & 7.19 & -0.3 & - & $*$ \\
\hline 2015/07/26 05:35:00 & 47.36 & 7.17 & -0.3 & - & $*$ \\
\hline 2015/07/26 06:42:55 & 47.36 & 7.12 & 2.5 & -1.0 & $*$ \\
\hline 2015/08/06 13:13:39 & 47.38 & 7.17 & -0.3 & - & $*$ \\
\hline 2015/08/09 09:41:43 & 47.32 & 7.08 & 2.3 & -0.4 & $*$ \\
\hline
\end{tabular}


Table 2 continued

\begin{tabular}{lllclll}
\hline Time & Lat & Lon & Depth $(\mathrm{km})$ & ML & \\
\hline 2015/08/11 11:55:23 & 47.47 & 6.91 & 1.8 & 1.7 & \\
2015/08/13 10:17:09 & 47.38 & 7.17 & -0.3 & -2.7 & $*$ \\
2015/08/14 00:27:27 & 47.35 & 7.17 & -0.3 & - & $*$ \\
2015/08/14 11:00:39 & 47.19 & 7.25 & 20.0 & 0.8 & \\
2015/08/15 00:53:29 & 47.44 & 7.37 & 15.2 & -0.2 & \\
2015/08/15 11:18:49 & 47.39 & 7.06 & 5.3 & -0.1 & $*$ \\
2015/08/18 13:40:28 & 47.35 & 7.18 & 0.5 & 0.7 & $* *$ \\
2015/08/28 09:10:03 & 47.44 & 7.41 & 13.2 & 1.8 & \\
\hline
\end{tabular}

The starred rows show the 86 events within a radius of $10 \mathrm{~km}$ around the laboratory. The rows marked with 2 stars indicate the events catalogued by the SED (Table 1)

trending, (2) SSE-dipping, (3) SSW-dipping, and (4) Subhorizontal. Each main group is composed of sub-groups based on their striation orientations. Another sub-group of synthetic focal mechanism (not shown in Fig. 4) is added to each main group by applying the regional stress orientation (defined in Fig. 5) to the respective fault orientation of the main groups.

\subsubsection{NNE-trending}

Based on the measurement of 19 fault orientations, this main group is trending $\mathrm{NNE} 015^{\circ}$ with an average dip direction of $42^{\circ}$. The orientation of these faults can be linked to the reactivation of NNE-SSW-striking normal faults associated with the Rhine Graben (Ustaszewski and Schmid 2007). On the basis of striation measurements, this main group can be further divided into sub-groups.

(a) NNE1

The NNE1 sub-group is based on 12 striation measurements with a plunge orientation of $149^{\circ} / 40^{\circ} \pm 12^{\circ}$. These orientations give a focal mechanism with a strike, dip, and rake of $018^{\circ} / 42^{\circ} / 043^{\circ}$. This focal mechanism represents a NNE-trending predominantly thrust-dominated fault system with a left-lateral strike-slip component.

\section{(b) NNE2}

The NNE2 sub-group is based on 7 striation measurements with a plunge orientation of $043^{\circ} / 6^{\circ} \pm 12^{\circ}$. These orientations give a synthetic focal mechanism with a strike, dip, and rake of $008^{\circ} / 34^{\circ} / 142^{\circ}$. This focal mechanism represents a NNE-trending predominantly thrust-related fault system with a right-lateral strike-slip component.

(c) NNEreg

If the average regional paleostress field based on the integration of the whole dataset $\left(\mathrm{SH}_{\max } \mathrm{NNW}\right)$ is applied to the NNE-trending fault orientation, we can derive a third synthetic focal mechanism (not shown in Fig. 4). This NNEreg sub-group has a focal mechanism orientation with a strike, dip, and rake of $015^{\circ} / 51^{\circ} / 052^{\circ}$. This focal mechanism represents a NNE-trending predominantly thrust-related fault system with a left-lateral strike-slip component similar to NNE1.

\subsubsection{SSE-dipping}

The 157 fault orientations of this main group show a general dip direction and dip of $156^{\circ} / 44^{\circ} \pm 2^{\circ}$. This orientation is parallel to the axis of the Mont Terri anticline (Laubscher 1977).

(a) $S S E$

The SSE-group, based on 157 fault/striation measurements is consistent with a synthetic focal mechanism with a strike, dip, and rake of $060^{\circ} / 44^{\circ} / 085^{\circ}$. This focal mechanism represents a SSE-dipping thrust-fault system.

(b) SSEreg

Applying $\mathrm{SH}_{\max } \mathrm{NNW}$ to the SSE-dipping faults, we calculate a synthetic focal mechanism with a strike, dip, and rake of $061^{\circ} / 43^{\circ} / 084^{\circ}$. This focal mechanism represents a SSE-dipping thrust fault, almost identical to the SSE group.

\subsubsection{SSW-dipping}

Based on the measurement of 32 fault orientations, we find a mainly SSW-dipping main group with a dip direction and dip of $199^{\circ} / 39^{\circ} \pm 6^{\circ}$. Here also we can differentiate several sub-groups.

(a) SSWI

The SSW1 sub-group is based on 16 striation measurements with a plunge orientation of $132^{\circ} / 15^{\circ} \pm 8^{\circ}$. These orientations yield a synthetic focal mechanism with a strike, dip, and rake of $112^{\circ} / 38^{\circ} / 157^{\circ}$. This focal mechanism represents a SSW-dipping predominantly thrust-related fault with a right-lateral strike-slip component.

(b) SSW2

The SSW2 sub-group is based on 9 striation measurements with a plunge orientation of $172^{\circ} / 9^{\circ} \pm 11^{\circ}$. These orientations give a synthetic focal mechanism with a strike, dip, and rake of $103^{\circ} / 41^{\circ} / 107^{\circ}$, and represents a SSW-dipping thrust fault.

(c) SSW3

The SSW3 sub-group is based on 7 striation measurements with a plunge orientation of $236^{\circ} / 5^{\circ} \pm 15^{\circ}$. These orientations yield a synthetic focal mechanism with a strike, dip, and rake of $109^{\circ} / 41^{\circ} / 060^{\circ}$, and represents a SSW-dipping predominantly thrust-related fault system with a left-lateral strike-slip component.

(d) SSWreg

Applying $\mathrm{SH}_{\max } \mathrm{NNW}$ to the SSW-dipping faults, we find a synthetic focal mechanism orientation with a strike, dip, and rake of $109^{\circ} / 40^{\circ} / 117^{\circ}$. This focal mechanism represents a SSE-dipping thrust fault similar to SSW2. 


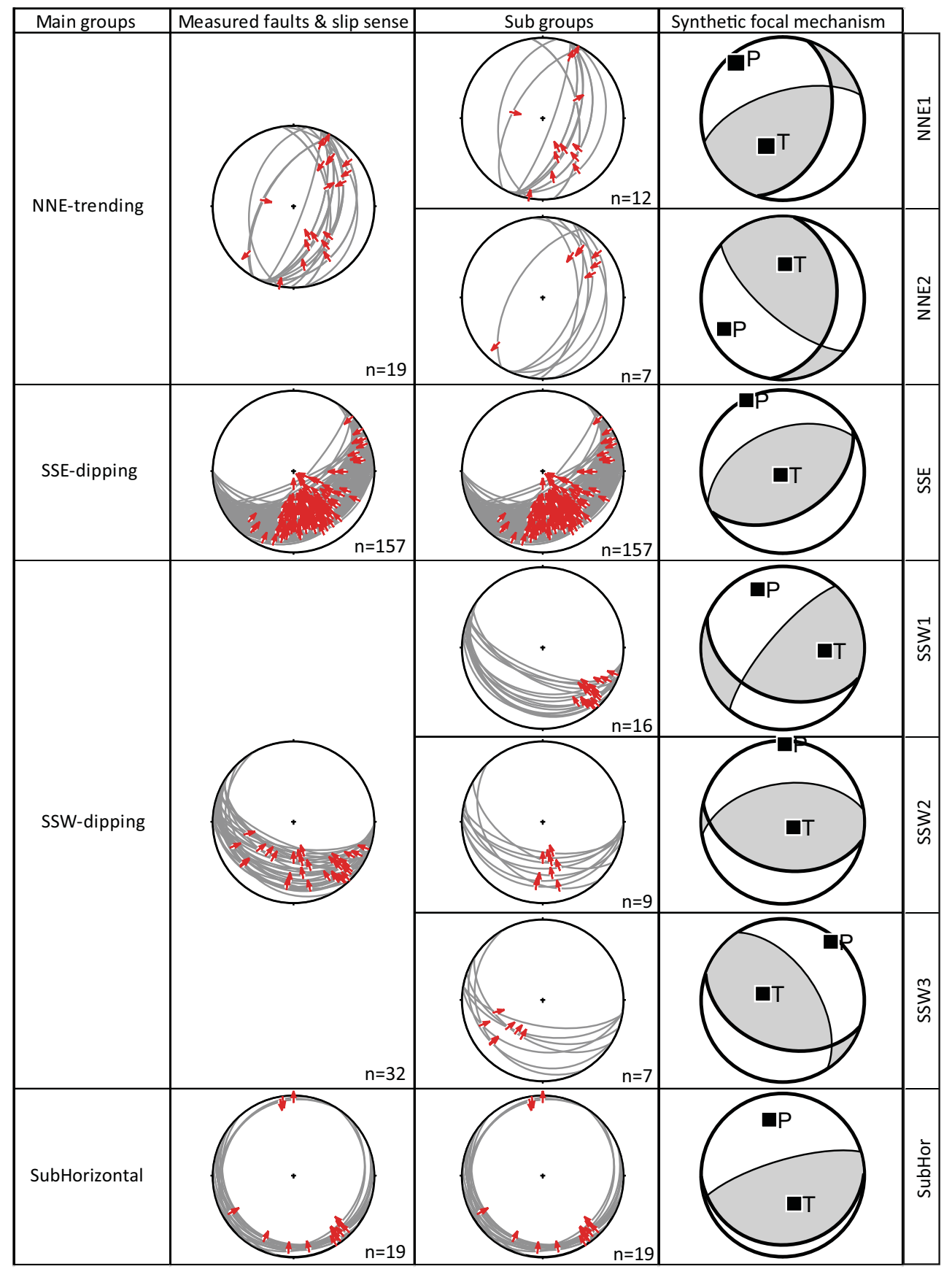

Fig. 4 Synthetic focal-mechanism main groups and sub-groups for microseismic events in the sedimentary cover. The orientation of 234 faults and striations in the sedimentary cover were measured during the excavation of the Mont Terri rock laboratory (Nussbaum et al. 2011). Out of a total dataset of 234 fault/striation pairs, 227 were used

\subsubsection{Subhorizontal}

A group of 19 subhorizontal faults has a general dip direction and $\operatorname{dip}$ of $179^{\circ} / 15^{\circ} \pm 4^{\circ}$. This orientation is parallel to the décollement surface at the basal Jura foldand-thrust belt (Laubscher 1997; Sommaruga 1999; Ustaszewski and Schmid 2007). to determine different main groups and sub-groups. These sub-groups determine the synthetic focal mechanisms composed of two nodal planes in which the bold great circle indicates the fault plane and the thin great circle shows the auxiliary plane

(a) SubHor

The SubHor group is based on 19 striation measurements with a plunge orientation of $179^{\circ} / 5^{\circ} \pm 4^{\circ}$. The synthetic focal mechanism with a strike, dip, and rake of $089^{\circ} / 15^{\circ} / 105^{\circ}$ and is related to a subhorizontal thrust fault. 
Fig. 5 Regional paleostressfield orientations using 234 measurements of fault orientations, striations, and slip sense in the Mont Terri rock laboratory (Nussbaum et al. 2011) plotted on a lower hemisphere stereoplot. The mean stress field $\left(\mathrm{SH}_{\max } \mathrm{NNW}\right)$ has an orientation of maximum horizontal compression towards the NNW with the P-axis $337^{\circ} \%$ $06^{\circ}$, T-axis $163^{\circ} / 84^{\circ}$, and $\mathrm{B}$-axis $067 \% 1^{\circ}$
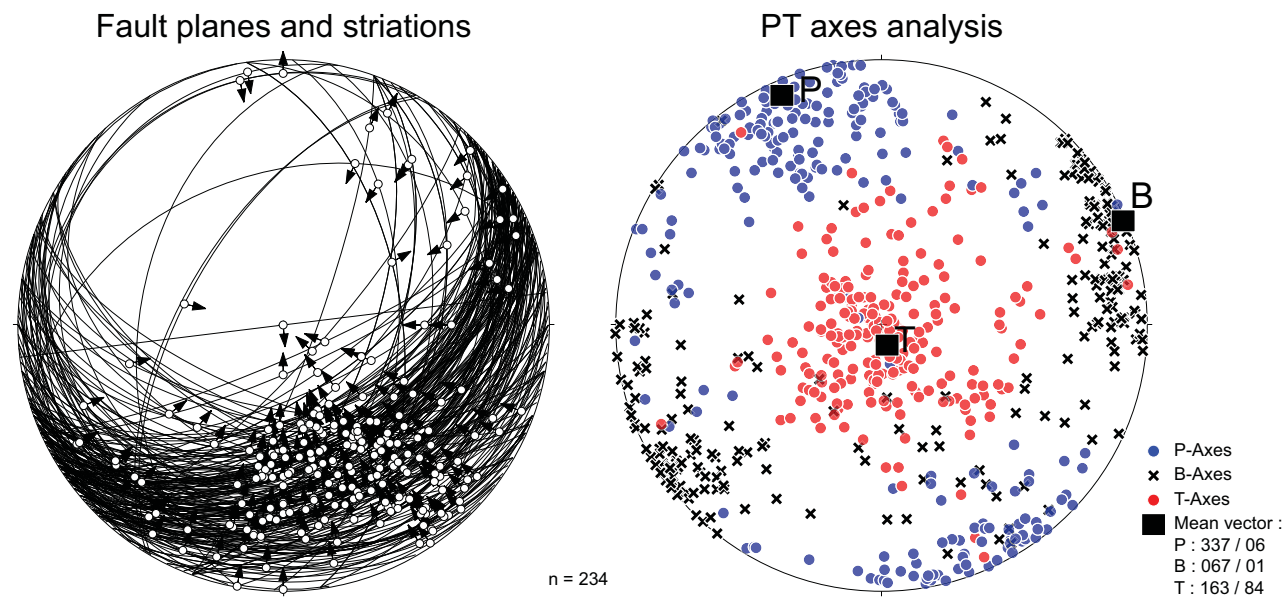

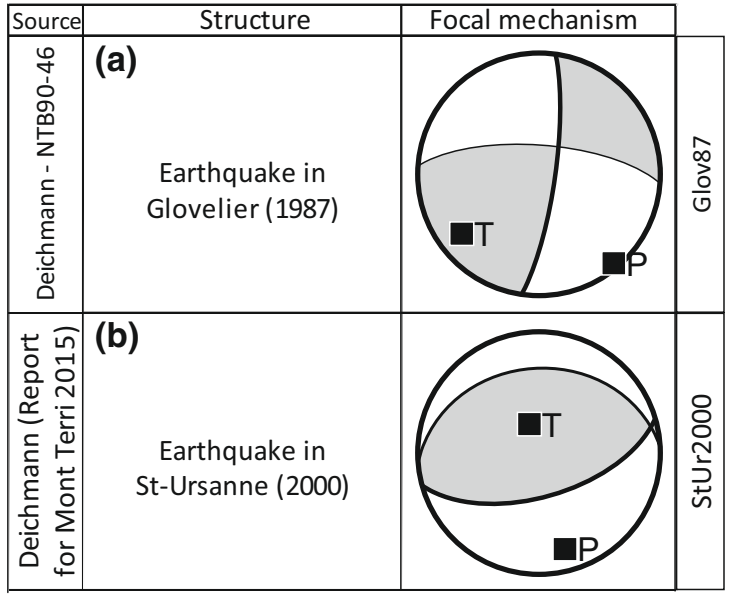

Fig. 6 Focal mechanisms and synthetic focal mechanisms for the microseismic events in the basement: a, b The focal mechanisms based on earthquakes analysed in Deichmann (1990), Baer et al. (2001), and Deichmann (2015). c, d Synthetic focal mechanisms

\section{(b) SubHorreg}

Applying $\mathrm{SH}_{\max } \mathrm{NNW}$ to the SubHor fault orientation yields a focal mechanism orientation with a strike, dip, and rake of $107^{\circ} / 11^{\circ} / 113^{\circ}$. This focal mechanism represents a subhorizontal thrust fault.

\subsection{Focal mechanisms from strong earthquakes in the basement}

One strong earthquake and one series of strong earthquakes, catalogued by the SED, were registered in the vicinity of the Mont Terri rock laboratory: (a) in Glovelier (1987) and (b) the series in St-Ursanne (2000). The hypocentre of these earthquakes is located in the basement beneath the main décollement.

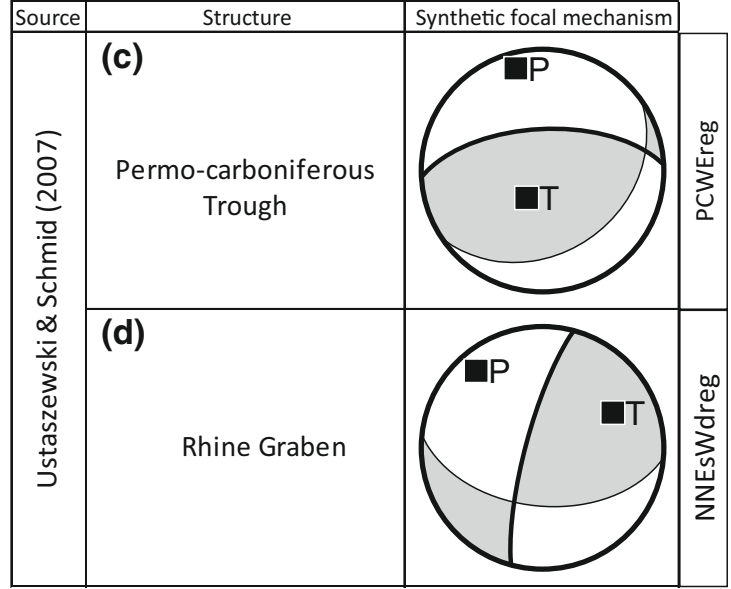

produced based on the orientation of interpreted faults by Ustaszewski and Schmid (2007) by applying the regional stress orientation (Fig. 5). The synthetic focal mechanisms have two nodal planes in which the bold great circle indicates the fault plane

\subsubsection{Glov87 and Glov87reg}

An earthquake of ML 3.7 occurred on December 11th 1987 02:25:58.2 (UTC) in Glovelier (Lat/Lon: $47.313^{\circ} \mathrm{N} /$ $\left.7.161^{\circ} \mathrm{E}\right)$ at a depth of $9 \mathrm{~km}$. The associated focal mechanism (Glov87) shows an orientation with a strike, dip, and rake of $008^{\circ} / 79^{\circ} / 020^{\circ}$ (Deichmann 1990), and represents a strike-slip fault. Depending on the nodal plane considered to be the fault plane, the fault mechanism is sinistral strikeslip on the NS-trending nodal plane, or dextral strike-slip on the W-E-trending nodal plane (Fig. 6).

If we apply $\mathrm{SH}_{\max } \mathrm{NNW}$ to the Glov87 fault orientation to calculate the Glov87reg focal mechanism, we obtain a strike, dip, and rake of $008^{\circ} / 79^{\circ} / 016^{\circ}$, which is almost identical to the present-day situation of Glov87. 


\subsubsection{StUr2000; StUr2000reg1; and StUr2000reg2}

A series of four earthquakes with a magnitude $\mathrm{ML} \leq 3.0$ occurred on March 28, 2000, and an earthquake of ML 3.2 on April 6, 2000 in St-Ursanne (Lat/Lon $47.367^{\circ} \mathrm{N} /$ $\left.7.168^{\circ} \mathrm{E}\right)$ at a depth of $1 \mathrm{~km}$. The focal mechanism orientation (StUr2000) has a strike, dip, and rake of $074 \% 65 \%$ $089^{\circ}$ if the SSE-dipping nodal plane is the fault plane, and $256^{\circ} / 25^{\circ} / 092^{\circ}$ if the NNW-dipping nodal plane is the fault plane (Deichmann 1990, 2015). This focal mechanism represents either a SSE-dipping steep reverse fault or a NNW-dipping back thrust (Fig. 6).

When applying $\mathrm{SH}_{\max } \mathrm{NNW}$ to the two nodal planes of the earthquake, we obtain two synthetic focal mechanisms differing by $30^{\circ}$ in orientation: StUr2000reg1 and StUr2000reg2. The focal mechanism StUr2000reg1 has an orientation of strike, dip, and rake of $266^{\circ} / 30^{\circ} / 098^{\circ}$, while the focal mechanism StUr2000reg2 has an orientation of strike, dip, and rake of $236^{\circ} / 31^{\circ} / 075^{\circ}$. These focal mechanisms (not shown in Fig. 6) have the same geological representation as the StUr2000 focal mechanism within a small variation of orientation.

\subsection{Synthetic focal mechanisms derived from published data on faults}

In the vicinity of the Mont Terri rock laboratory several structures indicating earthquake occurrences are known in the basement and can be related either to a Permo-Carboniferous trough or the Rhine Graben structure (Ustaszewski and Schmid 2007). We used these structures to apply the regional paleostress field $\mathrm{SH}_{\max } \mathrm{NNW}$ to derive synthetic focal mechanisms for each structure.

\subsubsection{PCWEreg}

Based on Ustaszewski and Schmid (2007), the PermoCarboniferous trough in northern Switzerland has an W-E orientation with a dip direction and dip of the border fault of $357^{\circ} / 02^{\circ}$. If we apply $\mathrm{SH}_{\max } \mathrm{NNW}$ to the WE-trending fault orientation this yields a PCWEreg synthetic focal mechanism with strike, dip, and rake of $056^{\circ} / 32^{\circ} / 063^{\circ}$. This represents a SSE-dipping sub-horizontal thrust fault or a NNW-dipping thrust fault (Fig. 6).

\subsubsection{NNEsWdreg}

Following Ustaszewski and Schmid (2007), the Rhine Graben structure is NNE-trending and dipping to the $\mathrm{W}$ $\left(285^{\circ} / 79^{\circ}\right)$. Applying $\mathrm{SH}_{\max } \mathrm{NNW}$ to the NNE-trending W-dipping fault orientation results in a focal mechanism with strike, dip, and rake of $195^{\circ} / 79^{\circ} / 041^{\circ}$ (NNEsWdreg), and a NNE-trending, left-lateral strike-slip component.

\subsubsection{Sub-horizontal}

Due to uncertainty of the hypocentral depth near the basement-cover interface, and because some focal mechanism solutions of seismicity in the basement suggest very shallow-dipping faults, we have added a group of subhorizontal faults to the basement groups (same as in the cover, see Fig. 4).

\section{Synthetic waveforms}

Microseismic events detected in the vicinity of the Mont Terri rock laboratory exhibit weak intensities and have been recorded by only a few stations near the source. It is, therefore, not possible to satisfactorily constrain a focal mechanism solution using first-motion analysis. An additional uncertainty arises from the interference of ambient noise with the signal of very weak events.

In order to overcome these pitfalls, we used synthetic waveforms in a wave-correlation approach to determine synthetic focal mechanisms for very weak events. Our procedure was as follows: we use synthetic focal mechanisms based on existing fault groups in the investigation area to generate synthetic waveforms that, subsequently, can be correlated with the recorded seismograms of the SNS and the Swiss permanent stations (Figs. 2,7) in the vicinity of the Mont Terri rock laboratory. The highest correlation factor between the naturally generated waveform and the synthetic waveforms represents the maximum likelihood estimate of the possible focal mechanism solution. The locations of the earthquakes from the microseismic catalog are further evaluated for their depth in relation to the geological context (see Freivogel and Huggenberger 2003; Nussbaum et al. 2017). Subsequently, we determine in which major geological unit (sedimentary cover or basement) each earthquake is located.

Groups of focal mechanisms that are in the sedimentary cover are identified through in situ measurements of fault orientations and striations. Another group of focal mechanisms is allocated to events arising in the basement. This latter group is composed of focal mechanisms from strong earthquakes that were located in the basement and synthetic focal mechanisms generated from structural interpretations of deep structures in the Mont Terri region based on data from the literature.

We generated synthetic waveforms for the stations near the source of the earthquake based on the synthetic focal mechanism defined from the corresponding groups of synthetic focal mechanisms. The waveforms were computed with WaveformTools, a modeling and moment-tensor inversion tool using an algorithm based on Green's functions 


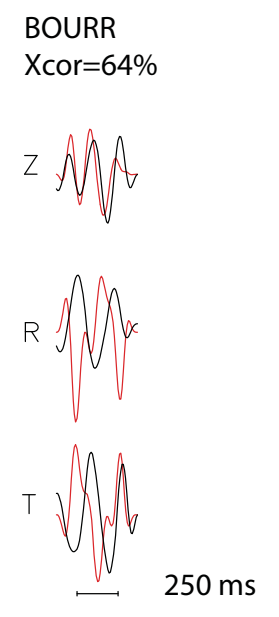

MTI02

Xcor $=75 \%$

Z
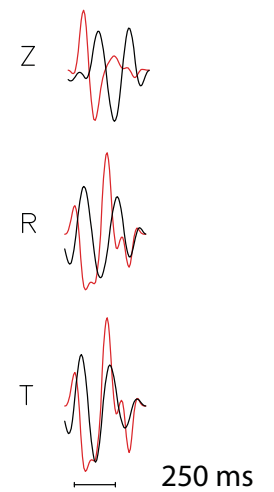

SNS1

Xcor $=75 \%$
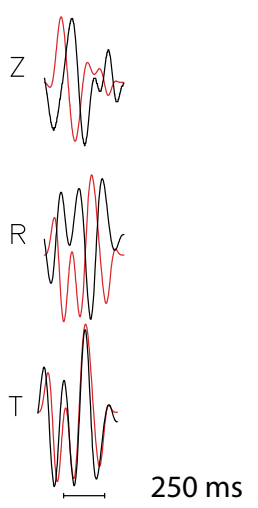

SNS2

Xcor $=45 \%$

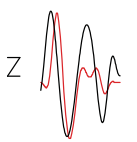

NNe

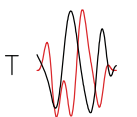

$\longmapsto \quad 250 \mathrm{~ms}$

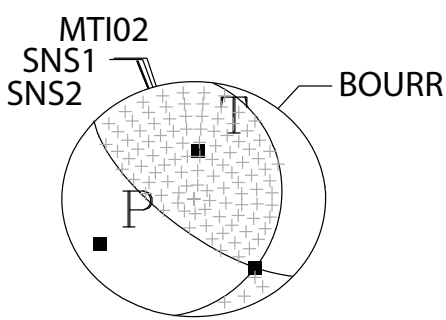

Fig. 7 Cross-correlation of recorded waveforms (black seismogram) with synthetic waveforms (red seismogram) at four stations generated from the synthetic focal mechanism group NNE2 (Fig. 4) for the

(Zeng and Anderson 1995; Ichinose et al. 2003; Helffrich et al. 2013). Fault orientations, direction, and sense of the movement are used as input to generate synthetic waveforms (see example in Fig. 7). The software also provides an algorithm for performing grid search to determine focal mechanisms based on waveform analysis. However, since the earthquakes that we considered evidenced very weak intensity, only the synthetic waveform generator algorithm is used to cross-correlate existing seismograms from surrounding stations.

We compute the waveforms and compare these using a $500 \mathrm{~ms}$ sampling window that contains the P-phase of the signal arriving at the stations in the vicinity of the Mont Terri rock laboratory. The sampling duration is chosen based on the average distance between the source of the earthquake and the stations, which has a complete waveform of the P-phase. The complete synthetic P-phase waveform is generated using the P-phase 3D velocity model of Switzerland (Husen et al. 2003).

\subsection{Layered velocity model}

To generate synthetic P-phase waveforms at the stations in the vicinity of the Mont Terri rock laboratory for seismogram correlation, a P-wave 1D-layered velocity model

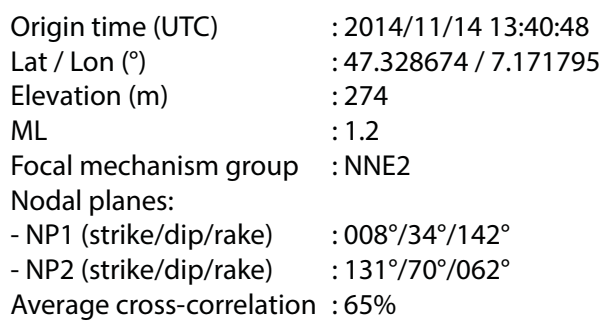

seismic event of 2014/11/14 13:40:48 (UTC) showing an average cross-correlation of $65 \%$

(Fig. 8) derived from a 3D velocity model of Switzerland (Husen et al. 2003) has been input into the WaveformTools software. Devising a more sophisticated and detailed local velocity model was beyond the scope of this pilot study. We therefore used the more regional, but robust, 3D velocity model of Switzerland (Husen et al. 2003) and applied the same parameters to all our dataset, being aware of the possibility of introducing errors due to changes in local geology and therefore in seismic velocity.

\subsection{Synthetic waveform correlations}

We correlated synthetic waveforms generated by the WaveformTools software with recorded seismograms from stations in the vicinity of the Mont Terri rock laboratory and applied these to 86 selected earthquakes (Table 2). The synthetic waveform correlation is done in two steps. The first step consists of performing an origin time scan based on the time given in the catalog (origin time and location) and the traveltime calculated by the synthetic waveform process depending on the respective layered velocity model. Due to the difference of methodology in locating earthquakes using Hypoline and the traveltime calculation using WaveformTools, a time lag of up to $2 \mathrm{~s}$ can occur. Based on the results of this first step, we could fit the origin 


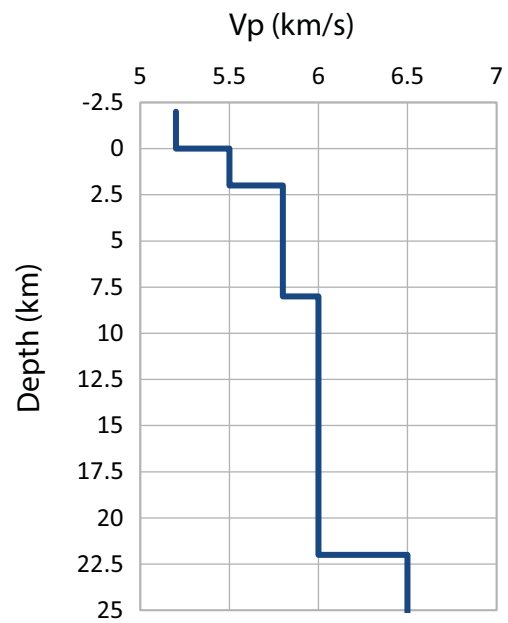

Fig. 8 1D P-wave velocity (Vp) model of Switzerland at the location of Mont Terri rock laboratory to the depth of $25 \mathrm{~km}$ derived from the 3D complex velocity model of Switzerland (Husen et al. 2003)

time to the maxima within $\pm 2 \mathrm{~s}$. We then input the origin time maxima obtained from the first step as the origin time for the second step.

The second step consists in calculating the correlation coefficient of the synthetic waveform compared with the seismogram for each station in the vicinity of the Mont Terri rock laboratory, based on the sub-groups of predefined focal mechanisms. We calculate the average correlation coefficient from the stations for a focal mechanism sub-group and chose the maximum average as the best-fit focal mechanism for the analyzed earthquake. A maximum average correlation coefficient below $50 \%$ was taken to be no result. Average correlation coefficients for waveforms of 86 earthquakes correlated with the synthetic waveforms from the seven groups of synthetic focal mechanisms derived from the different fault groups ranged from 50 to $71 \%$. In 7 instances the coefficient was $<50 \%$ and these were not considered further.

\section{Results}

Many earthquakes from the catalog correlated well with the NNE-trending synthetic focal mechanisms constructed from mapped and known faults. Their epicentres agree with NNE-trending faults represented on geological maps where these structures are correlated with the reactivation of preexisting normal faults related to the Rhine Graben (affecting both basement and/or cover). Hypocentres associated to the SSE-dipping focal mechanisms are also coherent with thrust faults of the anticline structure in the vicinity of the Mont Terri rock laboratory. The SSW-dipping focal mechanisms are associated with faults conjugate to the NNE-trending faults related to reactivation of the pre-existing NNE-SSW-striking Rhine Graben normal faults (Fig. 9).

To gain a better understanding on the seismotectonics related to structures in the vicinity of the Mont Terri rock laboratory, the focal mechanisms are represented as backsection hemisphere projection beachballs (Fig. 10) and displayed at their hypocentre depth on a geologic section (Fig. 1). Thus, based on our analysis it was possible to determine three synthetic focal mechanisms from the microseismic events near the Mont Terri rock laboratory. These are linked with the major thrust faults interpreted in the Mont Terri ramp-related anticline, especially with the basal ramp and the Main Fault known from the tunnel system (Nussbaum et al. 2017).

\section{Discussion}

In this pilot study we used 86 from a total of 198 recorded seismic events from the SED permanent network and data from a dedicated SNS network between July 2014 and August 2015, to improve the seismotectonic understanding of very weak seismicity, magnitude (ML) between -2.0 and 2.0, in the vicinity of the Mont Terri underground laboratory. Microseismicity in the detected magnitude range has a weak intensity, and therefore the seismic signal interferes with ambient noise. Thus, focal-mechanism inversion using first-motion analysis and a waveform grid search method results in solutions with large uncertainty. By correlating recorded seismograms with synthetic waveforms based on synthetic focal mechanism derived from fault orientation and striation measurements, we can associate a constructed (synthetic) focal mechanism to the weak-intensity microseismic events. This procedure is an affirmative methodology to associate existing in situ measured fault orientations and striations with detected very weak-intensity microseismicity in the vicinity of the Mont Terri rock laboratory.

Since the procedure works on the basis of known and in situ measured structures, the computational solution uncertainties that yield average correlation coefficients comparing synthetic to recorded waveforms ranging from 50 to $71 \%$ are considered qualified. These rather low values of correlation coefficients are primarily due to the weakintensity seismic signal interfering with ambient noise.

Being based on predefined (synthetic) focal mechanisms, our methodology does not allow us to investigate new or unknown structures. However, using map and section-view observations, we can correlate the location, orientation, and focal mechanism of microseismic events with the location and orientation of geological structures in the vicinity of the Mont Terri rock laboratory. The NNE-trending focal mechanisms of the microseismic 
4Fig. 9 Focal mechanisms of microseismic events in the vicinity of the Mont Terri rock laboratory represented as beachballs and fault orientations compared with known fault and thrust structures from the Geological Atlas of the Federal Office of Topography swisstopo. Two focal mechanisms determined by the Swiss Seismological Service (SED) are based on earthquakes analysed by Deichmann (1990, 2015) and Baer et al. (2001). The map is in the Swiss coordinate system CH1903

events are associated to existing NNE-SSW-striking mapped faults. We also observe that the SSW-dipping focal mechanisms are associated to conjugate faults with NNE-trending focal mechanisms. During the monitoring period, only a few events occurred that correlated with the SSE-dipping synthetic focal mechanisms. This synthetic focal mechanism could be related to thrusting of the Mont Terri anticline towards NNW. Thus on a regional scale, the synthetic focal mechanism solutions make it possible to use otherwise unconstrained seismic events to correlate with known structures in the basement and/or the cover.

\section{Suggestions for future application of our procedure}

To improve the results of focal mechanism attribution of a microseismic event, acquiring a complementary dataset of fault orientation and striation measurements in the vicinity of the Mont Terri rock laboratory would open the possibility to refine the orientation of the main group focal mechanism. Further, if we defined a more complex stress field with local/regional changes in the stress-field orientation and applied this to known fault orientations we could then better define the associated focal mechanism with an earthquake. Supervised and guided local grid search methods on the main groups of focal mechanism could also enable us to refine the determination of the focal mechanism of a seismic event. The WaveformTools software gives the possibility to attribute a specific velocity model for each station. This option could be used for further analysis to introduce local specific structure into the interpretation.
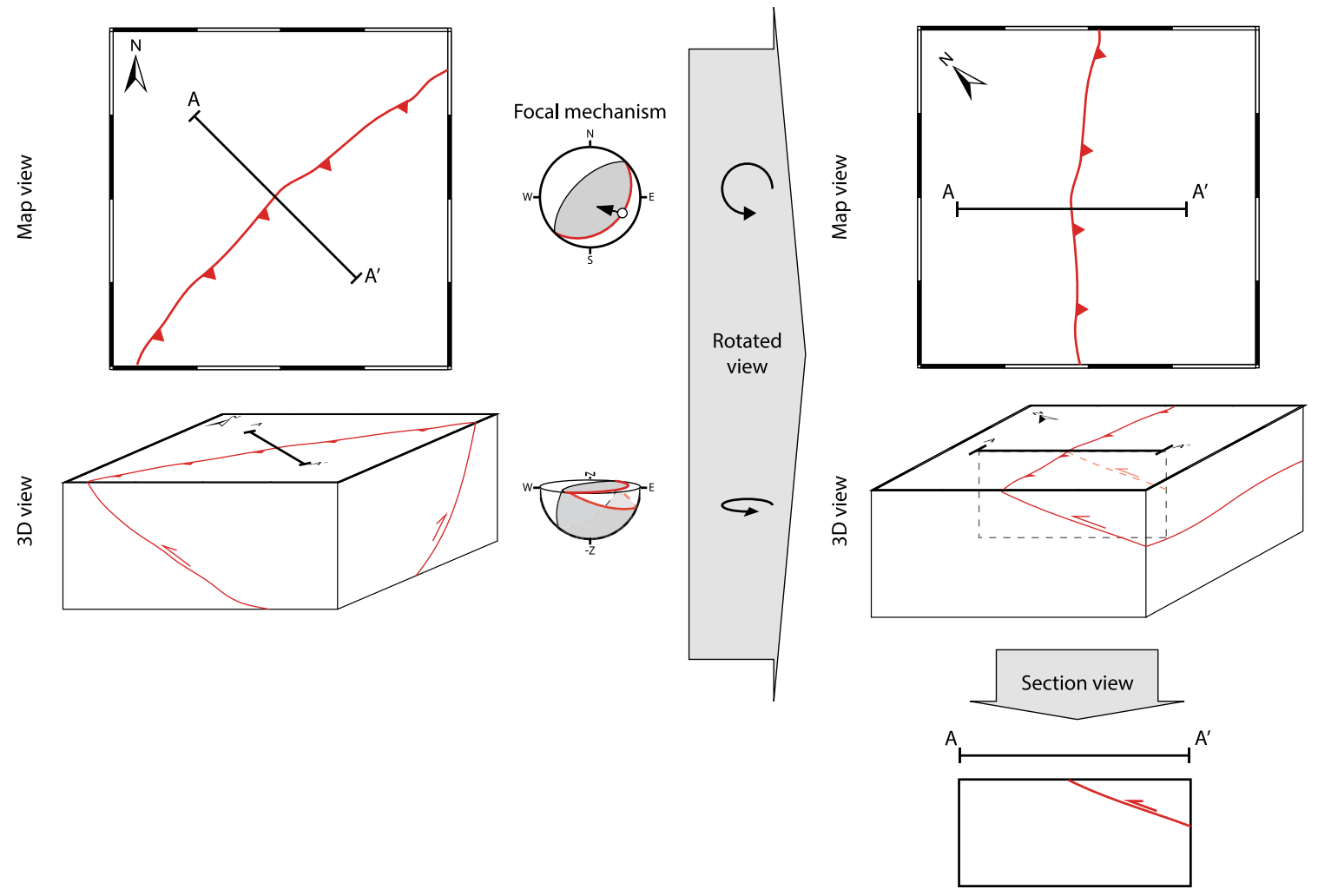

Focal mechanism
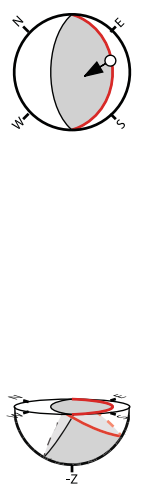

Back-section hemisphere projection focal mechanism

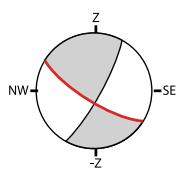

Fig. 10 Scheme for converting a lower-hemisphere projection focal mechanism in map view to a back-section-hemisphere projection focal mechanism in section view linked to the geometrical aspect of a fault 
Acknowledgements Thanks to the two reviewers, Prof. Guido Schreurs (Institute of Geological Sciences, University of Bern), and Dr. Marian Hertrich (Nagra), for significantly improving the manuscript. We also would like to thank Roy Freeman for helping with the English of the manuscript. UNIFR and swisstopo are thanked for financial support.

\section{References}

Aki, K., \& Richards, P. G. (1980). Quantitative seismology: Theory and methods (pp. 801-802). New York: W. H. Freeman and Co.

Allmendinger, R., Cardozo, N., \& Fisher, D. (2012). Structural geology algorithms: Vectors and tensors. Cambridge: Cambridge University Press.

Allmendinger, R. W., Gephart, J. W., \& Marrett, R. A. (1989a). Notes on fault slip analysis. Geol. Soc. Am. Short Course (pp. 66).

Allmendinger, R., Marret, R., \& Cladoulos, T. (1989b). Fault kinematics: a program for analysing fault slip data for Macintosh computer. Ithaca: Cornell University.

Baer, M., Deichmann, N., Braunmiller, J., Ballarin Dolfin, D., Bay, F., Bernardi, F., et al. (2001). Earthquakes in Switzerland and surrounding regions during 2000. Eclogae Geologicae Helvetiae, 94, 253-264.

Bossart, P., Bernier, F., Birkholzer, J., Bruggeman, C., Connolly, P., Dewonck, S., Fukaya, M., Herfort, M., Jensen, M., Matray, J-M., Mayor, J. C., Moeri, A., Oyama, T., Schuster, K., Shigeta, N., Vietor, T., Wieczorek, K.(2017). Mont Terri rock laboratory, 20 years of research: introduction, site characteristics and overview of experiments. Swiss Journal of Geosciences, 110. doi:10.1007/ s00015-016-0236-1 (this issue).

Cauzzi, C., \& Clinton, J. (2013). A high- and low-noise model for high-quality strong-motion accelerometer stations. Earthquake Spectra, 29(1), 85-102.

Deichmann, N. (1990). Seismizität der Nordschweiz, 1980-1988, und Auswertung der Erdbebenserien von Günsberg, Läufelfingen und Zeglingen. Nagra Technischer Bericht, 90-46. Nagra, Wettingen. http://www.nagra.ch.

Deichmann, N. (2015). Re-evaluation of the earthquake sequence of 2000 in the area of St-Ursanne and in the Mont Terri rock laboratory. Mont Terri Technical Note, TN 2013-99. Federal Office of Topography (swisstopo), Wabern, Switzerland. http:// www.mont-terri.ch.

Fäh, D., Giardini, D., Kästli, P., Deichmann, N., Gisler, M., SchwarzZanetti, G., Alvarez-Rubio, S., Sellami, S., Edwards, B., Allmann, B., Bethmann, F., Wössner, J., Gassner-Stamm, G., Fritsche, S., \& Eberhard, D. (2011). ECOS-09 Earthquake Catalogue of Switzerland Release 2011. Report and Database. Public catalogue, 17.4.2011. Report SED/RISK/R/001/20110417, Swiss Seismological Service ETH Zürich, Switzerland.

Freivogel, M., \& Huggenberger, P. (2003). Modellierung bilanzierter Profile im Gebiet Mont Terri-La Croix (Kanton Jura). In P. Heitzmann \& J.-P Tripet (Eds.), Mont Terri Project-Geology, paleohydrogeology and stress field of the Mont Terri region. Reports of the Federal Office for Water and Geology No. 4, (pp. 7-44). Federal Office of Topography (swisstopo), Wabern. http://www.mont-terri.ch.

Guglielmi, Y, Birkholzer, J., Rutqvist, J., Jeanne, P., \& Nussbaum, C. (2016). Can fault leakage occur before or without reactivation? Results from an in situ fault reactivation experiment at Mont Terri. In 13th International Conference on Greenhouse Gas Control Technologies, November 14-18, 2016, Lausanne.

Hardebeck, J. L., \& Shearer, P. M. (2002). A new method for determining first-motion focal mechanisms. Bulletin of the Seismological Society of America, 92(6), 2264-2276.
Heidbach, O., \& Reinecker, J. (2013). Analyse des rezenten Spannungsfelds der Nordschweiz. Nagra Arbeitsbericht (NAB 12-05). Nagra, Wettingen. http://www.nagra.ch.

Helffrich, G., Wookey, J., \& Bastow, I. (2013). The seismic analysis code: A primer and user's guide. Cambridge: Cambridge University Press.

Husen, S., Kissling, E., Deichmann, N., Wiemer, S., Giardini, D., \& Baer, M. (2003). Probabilistic earthquake location in complex three-dimensional velocity models: Application to Switzerland. Journal of Geophysical Research: Solid Earth, 108(B2).

Ichinose, G. A., Anderson, J. G., Smith, K. D., \& Zeng, Y. (2003). Source parameters of eastern California and western Nevada earthquakes from regional moment tensor inversion. Bulletin of the Seismological Society of America, 93(1), 61-84.

Joswig, M. (2008). Nanoseismic monitoring fills the gap between microseismic networks and passive seismic. First Break, 26, $121-128$.

Kobayashi, R., \& Nakanishi, I. (1994). Application of genetic algorithms to focal mechanism determination. Geophysical Research Letters, 21(8), 729-732.

Laubscher, H. (1977). Fold development in the Jura. Tectonophysics, 37, 337-362.

Laubscher, H. (1997). The décollement hypothesis of the Jura folding after 90 years. Bulletin of Applied Geology, 2(2), 167-182.

Marrett, R., \& Allmendinger, R. (1990). Kinematic analysis of faultslip data. Journal of Structural Geology, 12, 973-986.

NEA (1999). Geologic disposal of radioactive waste: Review of developments in the last decades. OECD Nuclear Energy Agency, Paris.

Nussbaum, C., Bossart, P., Amann, F., \& Aubourg, C. (2011). Analysis of tectonic structures and excavation induced fractures in the Opalinus Clay, Mont Terri underground rock laboratory (Switzerland). Swiss Journal of Geosciences, 104(2), 187-210.

Nussbaum, C., Kloppenburg, A., Caër, T., \& Bossart, P. (2017). Tectonic evolution around the Mont Terri rock laboratory, northwestern Swiss Jura: constraints from kinematic forward modelling. Swiss Journal of Geosciences. doi:10.1007/s00015016-0248-x (this issue)

Oppenheimer, D. H., Reasenberg, P. A., \& Simpson, R. W. (1988). Fault plane solutions for the 1984 Morgan Hill, California, Earthquake Sequence: Evidence for the state of stress on the Calaveras Fault. Journal of Geophysical Research: Solid Earth, 93, 9007-9026.

Plenefisch, T., \& Bonjer, K.-P. (1997). The stress field in the Rhine Graben area inferred from earthquake focal mechanisms and estimation of frictional parameters. Tectonophysics, 275(1), 71-97.

Sick, B., Walter, M., \& Joswig, M. (2012). Visual event screening of continuous seismic data by supersonograms. Pure and Applied Geophysics, 171, 549-559.

Sommaruga, A. (1999). Décollement tectonics in the Jura foreland fold-and-thrust belt. Marine and Petroleum Geology, 16, $111-134$.

Thury, M., \& Bossart, P. (1999). The Mont Terri rock laboratory, a new international research project in a Mesozoic shale formation, in Switzerland. Engineering Geology, 52, 347-359.

Ustaszewski, K., \& Schmid, S. (2007). Latest Pliocene to recent thick-skinned tectonics at the Upper Rhine Graben-Jura Mountains junction. Swiss Journal of Geosciences, 100(2), 293-312.

Vouillamoz, N., Wust-Bloch, G. H., Abednego, M., \& Mosar, J. (2016). Optimizing event detection and location in low-seismicity zones: case study from western Switzerland. Bulletin of the Seismological Society of America, 106(5), 2023-2036.

Woessner, J., \& Wiemer, S. (2005). Assessing the quality of earthquake catalogues: estimating the magnitude of 
completeness and its uncertainty. Bulletin of the Seismological Society of America, 95(2), 684-698.

Zeng, Y., \& Anderson, J. G. (1995). A method for direct computation of the differential seismogram with respect to the velocity change in a layered elastic solid. Bulletin of the Seismological Society of America, 85(1), 300-307.
Zoback, M. D., \& Harjes, H.-P. (1997). Injection-induced earthquakes and crustal stress at $9 \mathrm{~km}$ depth at the KTB deep drilling site, Germany. Journal of Geophysical Research: Solid Earth, 102(B8), 18477-18491. 